\title{
Bifluxon: Fluxon-Parity-Protected Superconducting Qubit
}

\author{
Konstantin Kalashnikov®, ${ }^{1, *}$ Wen Ting Hsieh $\odot,{ }^{2}$ Wenyuan Zhang $\odot,{ }^{1}$ Wen-Sen Lu, ${ }^{1}$ \\ Plamen Kamenov $\odot,{ }^{1}$ Agustin Di Paolo $\odot,{ }^{3}$ Alexandre Blais, ${ }^{3,4}$ Michael E. Gershenson $\odot,{ }^{1}$ and \\ Matthew Bell ${ }^{2, \dagger}$ \\ ${ }^{1}$ Department of Physics and Astronomy, Rutgers University, Piscataway, New Jersey, USA \\ ${ }^{2}$ Engineering Department, University of Massachusetts Boston, Boston, Massachusetts, USA \\ ${ }^{3}$ Institut Quantique and Département de Physique, Université de Sherbrooke, Sherbrooke, Quebec J1K 2RI, \\ Canada \\ ${ }^{4}$ Canadian Institute for Advanced Research, Toronto, Ontario, Canada
}

(Received 9 October 2019; accepted 12 August 2020; published 3 September 2020)

\begin{abstract}
We develop and characterize a symmetry-protected superconducting qubit that offers simultaneous exponential suppression of energy decay from charge and flux noises, and dephasing from flux noise. The qubit consists of a Cooper-pair box (CPB) shunted by a superinductor, forming a superconducting loop. Provided the offset charge on the CPB island is an odd number of electrons, the qubit potential corresponds to that of a $\cos (\phi / 2)$ Josephson element, preserving the parity of fluxons in the loop via Aharonov-Casher interference. In this regime, the logical-state wavefunctions reside in disjoint regions of Hilbert space, thereby ensuring protection against energy decay. By switching the protection on, we observe a tenfold increase of the decay time, reaching up to $100 \mu \mathrm{s}$. Though the qubit is sensitive to charge noise, the sensitivity is much reduced in comparison with the charge qubit, and the charge-noise-induced dephasing time of the current device exceeds $1 \mu \mathrm{s}$. Implementation of full dephasing protection can be achieved in the next-generation devices by combining several $\cos (\phi / 2)$ Josephson elements in a small array.
\end{abstract}

DOI: 10.1103/PRXQuantum.1.010307

\section{INTRODUCTION}

Superconducting qubits have emerged as one of the most promising platforms for quantum computing [1]. Over the past two decades, the coherence of these qubits has been improved by five orders of magnitude [2]. Even with this spectacular progress, implementation of error correction codes remains very challenging [3]. Further improvement in coherence will require the development of new approaches for mitigating harmful effects due to uncontrollable microscopic degrees of freedom, such as two-level systems (TLSs) in the qubit environment [4]. This route is provided by the improvement of materials for fabrication of superconducting qubits, which can lead to the reduction of the TLS density. A complementary approach, which we consider below, is based on the reduction of the qubit-TLS coupling by qubit design.

\footnotetext{
*kalashnikovkv@gmail.com

†Matthew.Bell@umb.edu

Published by the American Physical Society under the terms of the Creative Commons Attribution 4.0 International license. Further distribution of this work must maintain attribution to the author(s) and the published article's title, journal citation, and DOI.
}

Qubit coherence is characterized by the energy relaxation (decay) time $T_{1}$ and the dephasing time $T_{\varphi}$. The decay rate $\Gamma_{1} \equiv 1 / T_{1}$ due to coupling to a fluctuating quantity $\lambda$ is proportional to the transition amplitude $\left|\left\langle g\left|H_{\lambda}\right| e\right\rangle\right|^{2}$, where $H_{\lambda}$ is the coupling Hamiltonian and $\{|g\rangle,|e\rangle\}$ are the qubit's logical states. Since the external noise couples to local operators, decreasing of the overlap of $|g\rangle$ and $|e\rangle$ wavefunctions can significantly reduce $\Gamma_{1}$. This strategy is exploited by several qubit designs in which localization of the logical-state wavefunctions occurs within distinct and well-separated minima of the qubit potential, such as the "heavy fluxonium" qubit $[5,6]$.

On the other hand, a small dephasing rate $\Gamma_{\varphi} \equiv 1 / T_{\varphi}$ requires the qubit transition frequency $\omega_{g e}$ to be insensitive to fluctuations of $\lambda$. The first-order decoupling of a qubit from noise has been achieved at the so-called "sweet spot" $\lambda_{0}$, where $\partial \omega_{g e} /\left.\partial \lambda\right|_{\lambda_{0}}=0$ [7]. However, the coherence times achieved with this approach are insufficient for the implementation of the error correction codes, even if the drifts of the qubit operating point are eliminated over the timescale of operations. To remedy this, a "sweet-spot-everywhere" approach has been realized in the transmon qubit $[8,9]$ : an exponentially strong suppression of the qubit sensitivity to noise has been achieved by delocalization of the qubit wavefunctions in charge space. 
It is, however, worth noting that the two approaches of $T_{1}$ and $T_{\varphi}$ protection by qubit design come into conflict in the case of devices with a single degree of freedom in the qubit Hamiltonian (which we refer to as 1D qubits). For instance, at the dephasing sweet spot of the "heavy fluxonium" $[5,6]$ wavefunctions become delocalized due to its hybridization, which limits the decay time [Fig. 1(a), $i=1$ ], whereas $T_{1}$ protection can be realized only at the slope of the dispersion curve where $T_{\varphi}$ is small [Fig. 1(a), $i=2$ ]. In turn, the charge insensitivity of the transmon qubit is accompanied with strong dipole matrix elements that limit $T_{1}$ [Fig. 1(b), $\left.i=3,4\right]$. Additionally, the flatness of the transmon-qubit bands results in a strong reduction of the spectrum anharmonicity, potentially leading to a leakage of information outside of the computational subspace [10].

These examples suggest that a qubit Hamiltonian with full noise protection against relaxation and dephasing, i.e., exponentially large $T_{1}$ and $T_{\varphi}$, cannot be implemented in a single-mode superconducting quantum device. This conflict, however, can be reconciled by the so-called "fewbody" qubits [11] that incorporate more than one degree of freedom in the qubit Hamiltonian (the dimensionality $D>1)$ [12-15].

An example of simultaneous decay and dephasing protection in circuits with $D>1$ is given by the $0-\pi$ qubit [16]. Its $D=2$ Hamiltonian combines one "light" $\phi$ and one "heavy" $\theta$ variable. The logical wavefunctions are delocalized along the $\phi$ direction, while being localized

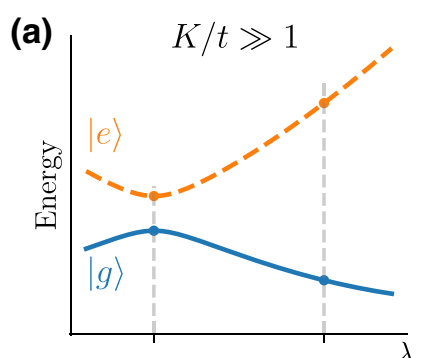

(1)

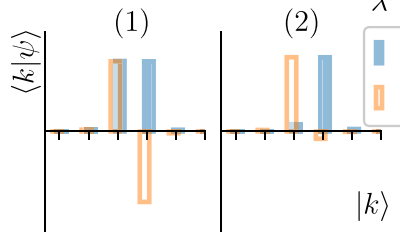

$|k\rangle$

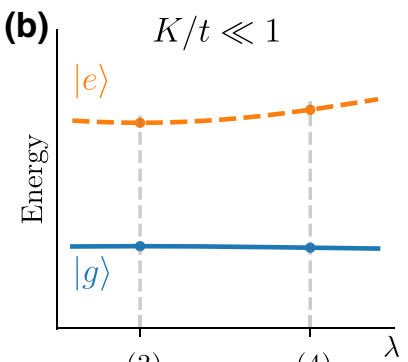

(4)

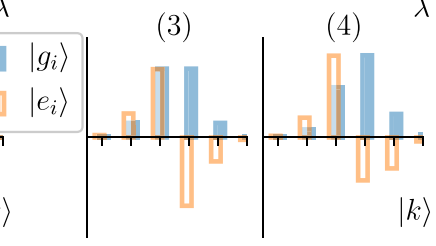

FIG. 1. The tradeoff between the decay and dephasing protection in superconducting qubits with a single charge or flux degree of freedom. The band structure (top panels) and wavefunctions (bottom panels) of a particle in quasiperiodic potentials: (a) the free-particle regime and (b) the tight-binding regime. The wavefunction overlap and the energy sensitivity $\partial E_{e g}^{(i)} / \partial \lambda$ do not simultaneously vanish for any point $(i)$. Flux (charge) qubits correspond to the case in which the control parameter $\lambda=\Phi_{\text {ext }}\left(q_{g}\right)$, kinetic energy $K=E_{L}\left(E_{C}\right)$, tunneling energy $t=E_{\text {sps }}\left(E_{J}\right)$, and $|k\rangle$ is a fluxon (charge) basis. in two disconnected potential wells labeled by $\theta=[0, \pi]$. These properties lead to exponentially reduced sensitivity to flux-noise fluctuations, i.e., negligible dephasing, and exponentially small matrix elements, i.e., long decay time [17]. Noise protection in this device is hardwired by circuit design, making the qubit robust against external perturbations. Fabricating such a circuit, however, entails several serious challenges, among which are very strict requirements on the parameters of all circuit elements and symmetry constraints. Moreover, since the built-in protection permanently decouples the qubit from the environment, new approaches to state preparation, qubit manipulation, and readout are required [18].

Another concept of qubit protection exploits symmetries of Hamiltonians with $D>1$ [19], an example being the qubit based on Josephson rhombi arrays [20], experimentally realized in Ref. [13]. In a single rhombus threaded by half of the magnetic flux quantum, the transport of individual Cooper pairs (CPs) is suppressed due to destructive Aharonov-Bohm interference, such that the rhombi chain supports correlated transport of CPs [i.e., acts as a $\cos (2 \phi)$ Josephson element]. The dephasing time of the qubit can be enhanced by delocalization of wavefunctions over the states with the same $\mathrm{CP}$ parity, which does not compromise $T_{1}$. Importantly, this qubit design enables on-demand switching of the qubit coupling to the environment (including the readout) on and off, which facilitates qubit manipulations. This also provides a route to fault-tolerant gates immune to noises in the control lines [21]. An improved version of the rhombus qubit can be built by parallel connection of several rhombi chains [22].

Here we focus on the implementation of a complementary circuit preserving the parity of fluxons in a superconducting loop, which consists of a split Cooper-pair box (CPB) and a superinductor (SI), and is depicted in Fig. 2(a). The probability of single-fluxon tunneling in and out of the loop can be tuned by the CPB charge $q_{g}$ of the CPB island (hereafter we refer to $\mathrm{CPB}$ charge modulo $2 e$ due to periodicity). At $q_{g}=1 e$ (where $e$ is the electron charge) Aharonov-Casher interference results in a $4 \pi$-periodic potential [i.e., $\cos (\phi / 2)$ Josephson element], which preserves the fluxon parity in the loop [23-25]. In the case of perfectly symmetric CPB junctions, the two degenerate logical states with different fluxon-number parity reside in disjoint regions of the Hilbert space, forbidding qubit decay. It is moreover possible to delocalize the wavefunction within each parity state via double fluxon tunneling in order to provide protection against pure dephasing by flux noise. Below we refer to such an element as a "bifluxon" qubit.

We design and characterize a prototype of the bifluxon qubit and demonstrate the decay protection by setting the $\mathrm{CPB}$ charge to the value of $1 e$. By turning protection on, we observe a tenfold increase of the decay time, up to 100 

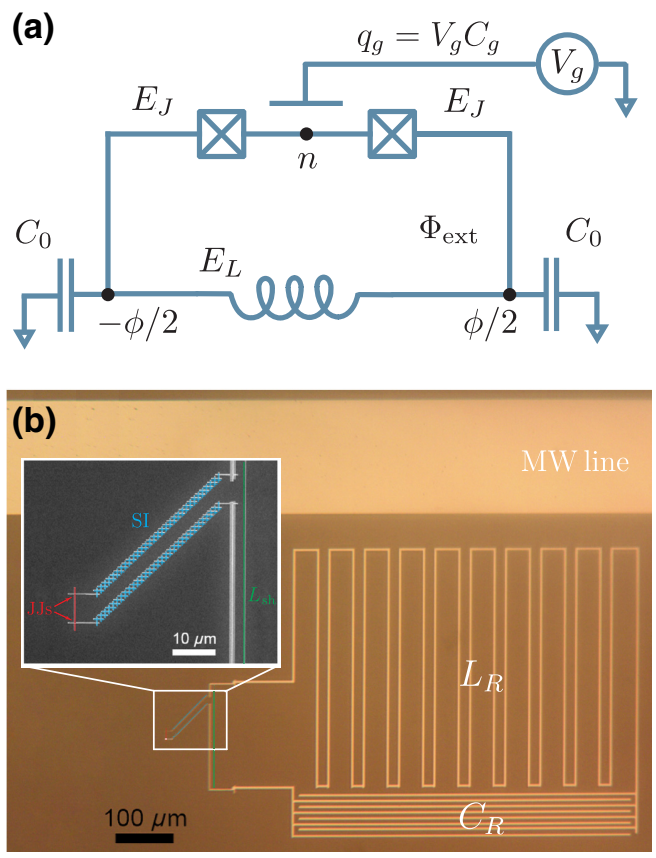

FIG. 2. (a) Simplified circuit scheme of the bifluxon qubit, described by Eq. (1). Charging energies of the superinductor and $\mathrm{CPB}$ are $E_{C L}$ and $E_{C}$, respectively. The qubit is controlled by the CPB charge $q_{g}$ and the magnetic flux $\Phi_{\text {ext }}$. (b) Optical image of the bifluxon qubit, readout resonator, and the microwave (MW) transmission line. The inset shows the SEM image of its central part: two JJs form the CPB island (red false color), the long array of larger JJs acts as a superinductor (blue), the narrow wire (green) forms the closed loop and couples the qubit to the readout resonator.

$\mu \mathrm{s}$. We also report the measurement of the qubit phasecoherence time $T_{\varphi}$ exceeding $1 \mu \mathrm{s}$.

The paper is organized as follows. In Sec. II we elaborate on the coherence properties of the bifluxon qubit by analyzing the symmetries of the logical wavefunctions and the resulting selection rules, as well as possible ways to realize dephasing protection. In Sec. III we present experimental implementation of the bifluxon qubit and discuss coherence-time measurement protocols. In Sec. IV we analyze the coherence limitations of the bifluxon qubit, and discuss a number of possibilities for further coherence-time improvements.

\section{THEORY}

In this section we outline the theory of the bifluxon qubit and the origin of its noise protection. We assume for simplicity that the Josephson junctions (JJs) forming the CPB are identical with Josephson energy $E_{J}=\Phi_{0} I_{0} / 2 \pi$, where $I_{0}$ is the JJ critical current and $\Phi_{0}=h / 2 e$ is the quantum of magnetic flux. The SI inductance and inductive energy are $L$ and $E_{L}=\left(\Phi_{0} / 2 \pi\right)^{2} / L$, respectively. The physical implementation of the SI will be considered in the next section.
The behavior of the system is determined by two controllable parameters: the offset charge $q_{g}$ of the CPB island and the external flux $\Phi_{\text {ext }}$ through the device's loop. Below we use the dimensionless quantities $\varphi_{\text {ext }}=2 \pi \Phi_{\text {ext }} / \Phi_{0}$ and $n_{g}=q_{g} / 2 e$. The full circuit Hamiltonian has three degrees of freedom (see Appendix A): the superconducting phase $\varphi$ of the CPB island and the sum and difference of the phases at the ends of the superinductor, respectively denoted by $\phi_{+}$and $\phi$. For simplicity, we assume that the high-frequency circuit mode $\phi_{+}$is not excited. Under this approximation, the qubit Hamiltonian is two dimensional, the kinetic energy scales of $\varphi$ and $\phi$ are denoted by $E_{C}$ and $E_{C L}$ and can be seen as CPB and SI charging energies, correspondingly. In the charge basis for the CPB degree of freedom, the circuit Hamiltonian can be written as

$$
\begin{aligned}
H= & \sum_{n}\left[4 E_{C}\left(n-n_{g}\right)^{2}|n\rangle\langle n|-E_{J} \cos \left(\frac{\phi}{2}\right)\left(\sigma_{n}^{+}+\sigma_{n}^{-}\right)\right] \\
& -4 E_{C L} \partial_{\phi}^{2}+\frac{E_{L}}{2}\left(\phi-\varphi_{\mathrm{ext}}\right)^{2},
\end{aligned}
$$

where $n$ represents the number of Cooper pairs in the CPB island, and we have defined $\sigma_{n}^{+}=|n+1\rangle\langle n|$ and $\sigma_{n}^{-}=\left(\sigma_{n}^{+}\right)^{\dagger}$.

To illustrate the working principles of the bifluxon qubit, here we examine the limiting case $E_{C} \gg E_{J}$, although full numerical diagonalization is used to analyze the data from devices with $E_{J} \gtrsim E_{C}$ below. Let us consider two cases for the offset charge $n_{g}$. If $n_{g}$ is set near an integer number $N$, the CPB degree of freedom can be thought of as "frozen" close to the charge state that minimizes the kinetic-energy term in Eq. (1). In this case, the circuit Hamiltonian is reduced to a one-dimensional fluxoniumlike Hamiltonian with a renormalized Josephson energy $E_{J}^{2} / 4 E_{C}$ (see Appendix B). To operate the bifluxon qubit in the protected regime, the offset charge instead should be set close to halfinteger, i.e., $n_{g} \approx \frac{1}{2}$. With $E_{C} \gg E_{J}$, it is sufficient to consider only two nearly degenerate $\mathrm{CPB}$ states $|0\rangle$ and $|1\rangle$. Projecting the circuit Hamiltonian in this two-dimensional subspace, we find that

$$
\begin{aligned}
H_{r}= & 4 E_{C}\left(\frac{1}{2}-n_{g}\right) \sigma^{z}-E_{J} \cos \left(\frac{\phi}{2}\right) \sigma^{x} \\
& -4 E_{C L} \partial_{\phi}^{2}+\frac{E_{L}}{2}\left(\phi-\varphi_{\mathrm{ext}}\right)^{2},
\end{aligned}
$$

where $\sigma^{z}=|1\rangle\langle 1|-| 0\rangle\langle 0|$ and $\sigma^{x}=\sigma^{+}+\sigma^{-}$. Equation (2) is diagonal in the $\sigma^{x}$ basis for $n_{g}=\frac{1}{2}$. Therefore, the lowest-energy eigenstates can be factorized as $\left|\psi^{n}\right\rangle \otimes$ $\left|\psi^{\phi}\right\rangle$, where the superscripts $n$ and $\phi$ denote the charge-like and flux-like components of the wavefunctions, respectively. In particular, the charge-like component results in either the symmetric or antisymmetric combinations $\left| \pm^{n}\right\rangle=(|0\rangle \pm|1\rangle) / \sqrt{2}$. The flux-like component is an 
(a)
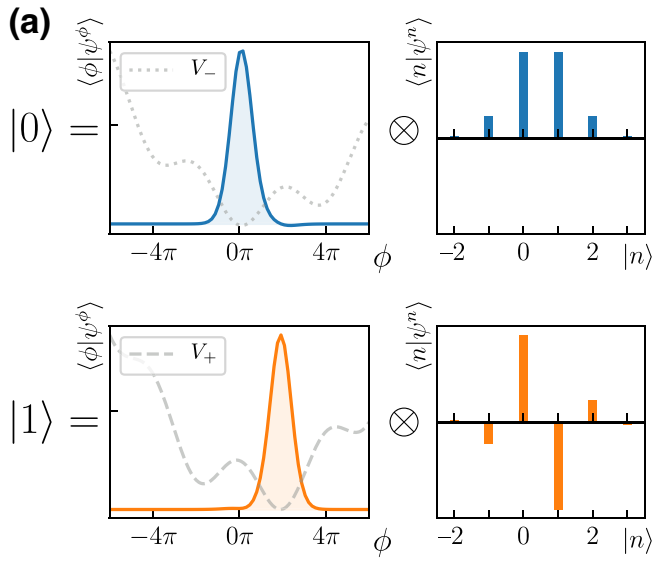

(b)

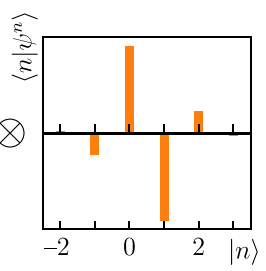

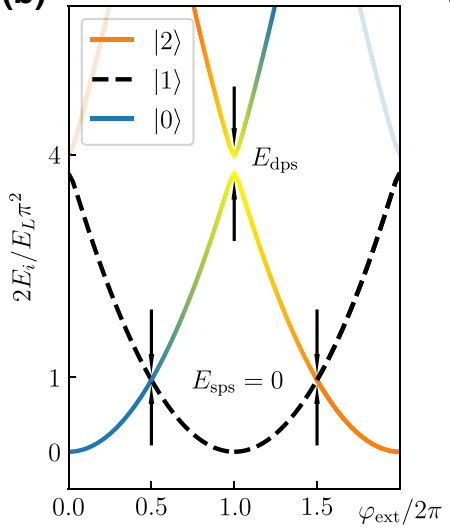

(c)
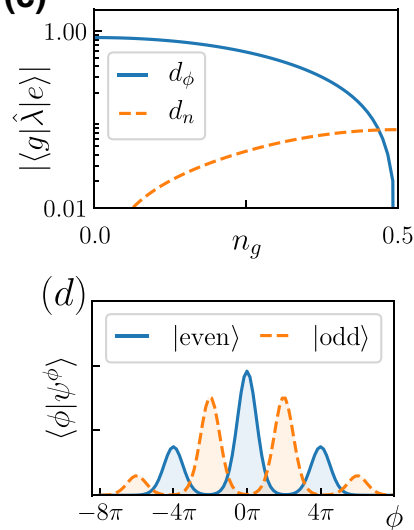

FIG. 3. (a) The ground and first excited states of the bifluxon qubit shown as products of the fluxon wavefunctions in the $V_{ \pm}$ potentials and CPB state for $\varphi_{\mathrm{ext}}=\pi, n_{g}=0.5$. The parity of the $\cos (\phi / 2)$ term is controlled by the CPB state $\left| \pm^{n}\right\rangle$. (b) Bifluxon energy bands as a function of $\varphi_{\text {ext }}$ at $n_{g}=0.5$. Color gradient represents the hybridization of the states with different fluxon numbers. Note the crossing of the parabolas at halfinteger $\varphi_{\mathrm{ext}} / 2 \pi$ due to $E_{\mathrm{sps}}=0$ and the avoided crossing between the next-to-neighbor parabolas $E_{\mathrm{dps}}>0$. (c) Dependence of the flux and charge matrix elements on the CPB charge at $\varphi_{\mathrm{ext}}=0$. The decay protection is realized at $n_{g}=0.5$, where $d_{\phi}$ is zero and $d_{n}$ is significantly suppressed. (d) Delocalization of the even (odd) fluxon states in the regime $E_{\mathrm{dps}} \gg 2 \pi^{2} E_{L}$ leads to suppression of dephasing due to the flux noise. Wavefunctions are calculated for $\varphi_{\mathrm{ext}}=0, n_{g}=0.5$..

eigenstate of the one-dimensional Hamiltonian $H_{ \pm}=$ $-4 E_{C L} \partial_{\phi}^{2}+V_{ \pm}$, with a potential energy that depends on the charge state

$$
V_{ \pm}=\mp E_{J} \cos \left(\frac{\phi}{2}\right)+\frac{E_{L}}{2}\left(\phi-\varphi_{\mathrm{ext}}\right)^{2} .
$$

The local minima of the fluxonium-like potential $V_{+}\left(V_{-}\right)$ are positioned near $\phi_{m}=2 \pi m$, where $m$ is an even (odd) integer. A harmonic-oscillator wavefunction of the form $\psi_{m}(\phi) \sim \exp \left[-\sqrt{E_{J} / E_{C L}}\left(\phi-\phi_{m}\right)^{2} / 4\right]$, localized at the $m$ th minimum, can be associated with a fluxon excitation $|m\rangle$. Using the fluxon representation, the eigenstates of Eq. (2) can be expressed as $|m\rangle=\{|2 k\rangle\} \cup\{|2 k+1\rangle\}$, where $|2 k\rangle=\left|+^{n}, \psi_{2 k}^{\phi}\right\rangle$ and $|2 k+1\rangle=\left|-^{n}, \psi_{2 k+1}^{\phi}\right\rangle$ have an even and odd number of fluxons in the loop, respectively [see Fig. 3(a)].

In Fig. 3(b) we present the spectrum of the qubit for $n_{g}=\frac{1}{2}$ as a function of $\varphi_{\text {ext }}$. Since the single phase-slip (SPS) processes connecting $|m\rangle \leftrightarrow|m+1\rangle$ are forbidden due to the symmetry of the wavefunctions

$$
E_{\mathrm{sps}}=\left\langle m\left|H_{r}\right| m+1\right\rangle \propto\left\langle+{ }^{n} \mid-{ }^{n}\right\rangle=0,
$$

the two neighboring parabolas cross at halfinteger $\varphi_{\text {ext }} / 2 \pi$. This can be interpreted as a fluxon-parity conservation rule due to the Aharonov-Casher effect [23], which has been experimentally observed in Refs. [24-26]. Therefore, at a halfinteger $n_{g}$, the considered system resembles a fluxonium qubit made up of a $4 \pi$-periodic Josephson element, justifying the name "bifluxon."

Double phase-slip (DPS) processes mix fluxon states with the same parity ( $m$ and $m+2$ ), opening energy gaps in the spectrum. The DPS amplitude is given by

$$
\begin{aligned}
E_{\mathrm{dps}} & =\left\langle m\left|H_{r}\right| m+2\right\rangle \\
& =\hbar \omega_{p}\left\langle\psi_{m}^{\phi} \mid \psi_{m+2}^{\phi}\right\rangle \\
& \approx \hbar \omega_{p} \exp \left(-\pi^{2} \beta\right),
\end{aligned}
$$

where $\omega_{p}=\sqrt{8 E_{J} E_{C L}}$ is a plasma frequency for the $V_{ \pm}$ potentials and $\beta=\sqrt{2 E_{J} / E_{C L}}$.

The symmetry of states with distinct fluxon parity makes the qubit immune to energy decay due to both flux and charge noises. Indeed, the phase dipole-moment matrix element is identically zero,

$$
d_{\phi} \sim\langle m|\hat{\phi}| m+1\rangle \propto\left\langle+{ }^{n} \mid-{ }^{n}\right\rangle=0,
$$

whereas, provided $E_{J} \gg E_{C L}$, the matrix element of the charge-noise operator is exponentially suppressed in comparison with the charge qubit [27],

$$
d_{n} \sim\left\langle m\left|\sigma^{z}\right| m+1\right\rangle=\left\langle\psi_{m}^{\phi} \mid \psi_{m+1}^{\phi}\right\rangle=\exp \left(-\pi^{2} \beta / 4\right) .
$$

Deviations from the optimal point $n_{g}=0.5$ lead to protection degradation. In the first order of perturbation theory, the phase matrix element increases linearly with $\delta n=$ $n_{g}-0.5$, but remains exponentially small in $\beta$ :

$$
d_{\phi}(\delta n) \sim E_{C} / E_{L} \exp \left(-\pi^{2} \beta / 4\right) \delta n .
$$

In Fig. 3(c) we show the charge and phase dipole-moment matrix elements obtained by numerical diagonalization of the full Hamiltonian Eq. (1). The weak sensitivity to 
charge noise is comparable to the flux sensitivity of a heavy fluxonium $[5,6]$, and can be suppressed by stronger localization of the single-well excitations within the $V_{ \pm}$ potential minima by increasing the $E_{J} / E_{C L}$ ratio.

The decay protection due to symmetries of the bifluxoncircuit wavefunctions can also be understood in the following way. Consider a logical qubit made of two faulty qubits labeled $\rho$ and $\tau$, with the two lowest-energy states $|g\rangle=\left|\uparrow^{\rho} \downarrow^{\tau}\right\rangle$ and $|e\rangle=\left|\downarrow^{\rho} \uparrow^{\tau}\right\rangle$ separated from the others by a sizeable energy gap $\Delta E$. Since uncorrelated fluctuations of $\rho$ and $\tau$ cannot induce $g \leftrightarrow e$ transition and the leakage out of computation space is penalized by $\Delta E$, the qubit is protected against local noise in the $\rho$ and $\tau$ subsystems. Accordingly, the bifluxon qubit is protected against decay due to uncorrelated charge and flux noises.

In addition to decay protection, the bifluxon qubit can also be robust to flux-noise dephasing. Indeed, similarly to the case of the fluxonium qubit, the flux dispersion of the qubit can be reduced by increasing the superinductance value. This enables wider delocalization of the qubit wavefunctions in disjoint subspaces with different fluxon parities, as shown in Fig. 3(d). Quantitatively, the wavefunctions spread out over $G \approx \sqrt{E_{\mathrm{dps}} / E_{L}}$ potential wells. The flux dispersion is then suppressed by a factor of $\exp (-G)$ for $G \gg 1$. Therefore, the bifluxon qubit becomes exponentially insensitive to flux-noise dephasing under the condition

$$
E_{\mathrm{dps}} \gg 2 \pi^{2} E_{L} .
$$

It is important to note that the parameter $\beta$ controls the degree of localization of the $\psi_{m}(\phi)$ wavefunctions in the $m$ th potential minima. Stronger localization associated with large $\beta$ values results in (a) desirable suppression of the sensitivity to charge noise $\left(\left\langle\psi_{m}^{\phi} \mid \psi_{m+1}^{\phi}\right\rangle \rightarrow\right.$ 0 ), and (b) undesirable reduction of the double-phase slip rate $E_{\mathrm{dps}}$ and the quantum fluctuations of the phase $\left(\left\langle\psi_{m}^{\phi} \mid \psi_{m+2}^{\phi}\right\rangle \rightarrow 0\right.$ as well). Simultaneous fulfillment of the requirements $d_{n}^{2} \ll 1$ and $E_{\mathrm{dps}} \gg 2 \pi^{2} E_{L}$ is challenging, provided the self-resonance frequency of the SI, $f_{\text {sr, }}$, should be much higher than the qubit resonance frequency. However, recent progress in fabrication of ultrahigh-inductance elements with impedance $Z=$ $2 \pi f_{\mathrm{sr}} L>200 \mathrm{k} \Omega$ is encouraging. To reduce the chargenoise sensitivity by an order of magnitude $\left(d_{n}^{2}=0.1\right)$, the double-phase rate should not exceed $E_{\mathrm{dps}}=0.01 \times \hbar \omega_{p} \simeq$ $h \times 0.5 \mathrm{GHz}$ for a typical value of the plasma frequency $\omega_{p} / 2 \pi=50 \mathrm{GHz}$. The marginally required superinductor energy $E_{L} \simeq E_{\mathrm{dps}} / 2 \pi^{2} \simeq h \times 20 \mathrm{MHz}$ is only three times smaller than the value of $h \times 65 \mathrm{MHz}$ that has already been experimentally realized [28].

Finally, it should be noted that, since the bifluxon is inherently a charge-sensitive device, a single qubit does not offer protection against the charge-noise-induced dephasing. As we discuss in Sec. IV, a small array of such elements can in principle provide a polynomial increase of the dephasing time and help to overcome this limitation.

\section{EXPERIMENT}

In this work, the bifluxon qubit is realized as a splitjunction CPB [a superconducting island flanked by two small nominally identical JJs with Josephson and charging energies $E_{J}$ and $E_{C J}$, respectively; see Fig. 2(b)] shunted by a SI, which is implemented as an array of $N_{A}=122$ larger JJs with corresponding energies $E_{J A}$ and $E_{C A}$. The sizes of small $\left(0.11 \times 0.16 \mu \mathrm{m}^{2}\right)$ and large $\left(0.21 \times 0.30 \mu \mathrm{m}^{2}\right)$ junctions are chosen in order to allow phase-slip events across the CPB junctions $\left(E_{J} / E_{C J} \sim 1\right)$, but suppress the phase slips in the array $\left(E_{J A} / E_{C A} \gg 1\right)$. As long as the inductive energy of the SI chain $E_{L}=E_{J A} / N_{A}$ is much smaller than $E_{J}$, the phase across the SI is close to an integer number of $2 \pi$. The stray capacitance of the superconducting islands to the ground in combination with the junction capacitances results in charging energies $E_{C}$ and $E_{C L}$ of the CPB and the SI, respectively (see Appendix A for details). The self-resonant mode of the SI with frequency $f_{\text {sr }}$, determined by the SI inductance and its stray capacitance to the ground $C_{0}$, should remain well above the qubit transition frequency (usually a few gigahertz) in order to avoid qubit coupling to this mode.

The bifluxon qubit is controlled by the magnetic flux in the loop $\Phi_{\text {ext }}$ and the offset charge $q_{g}$, induced by applying the dc bias voltage to the coupling capacitor $C_{g}$ between the microstrip line and the CPB island. In order to perform the dispersive measurements of the bifluxon qubit, the device is inductively coupled to a lumpedelement readout resonator with capacitance $C_{R}=120 \mathrm{fF}$ and inductance $L_{R}=4 \mathrm{nH}$. For the coupling, a portion of the bifluxon superconducting loop with kinetic inductance $L_{\mathrm{sh}}=0.4 \mathrm{nH}$ is shared with the readout resonator. The qubit-resonator coupling constant for the device described in this paper is $g / 2 \pi=52 \mathrm{MHz}$. The full list of experimental parameters is provided in Appendix D.

In the transmission measurements, the microwave signals travel along the microstrip line that is coupled to the readout resonators of up to five different bifluxon qubits measured in the same cooldown. The qubits are individually addressed due to different resonant frequencies of the readout resonators. The bifluxon qubit, readout resonator, and microstrip transmission lines are fabricated in a single multiangle electron-beam deposition of aluminum through a liftoff mask (for fabrication and measurement details, see Refs. [13,24]).

The pump tone $f_{p}$ induces the $|0\rangle-|i\rangle$ transitions at the resonance frequencies $f_{0 i}=\left(E_{i}-E_{0}\right) / h$. The measurement tone $f_{m}$ probes the dispersive shift of the coupled readout resonator. Although the dispersive measurements in the protected regime are complicated by significantly reduced qubit-readout coupling, the signal-to-noise 
(a)
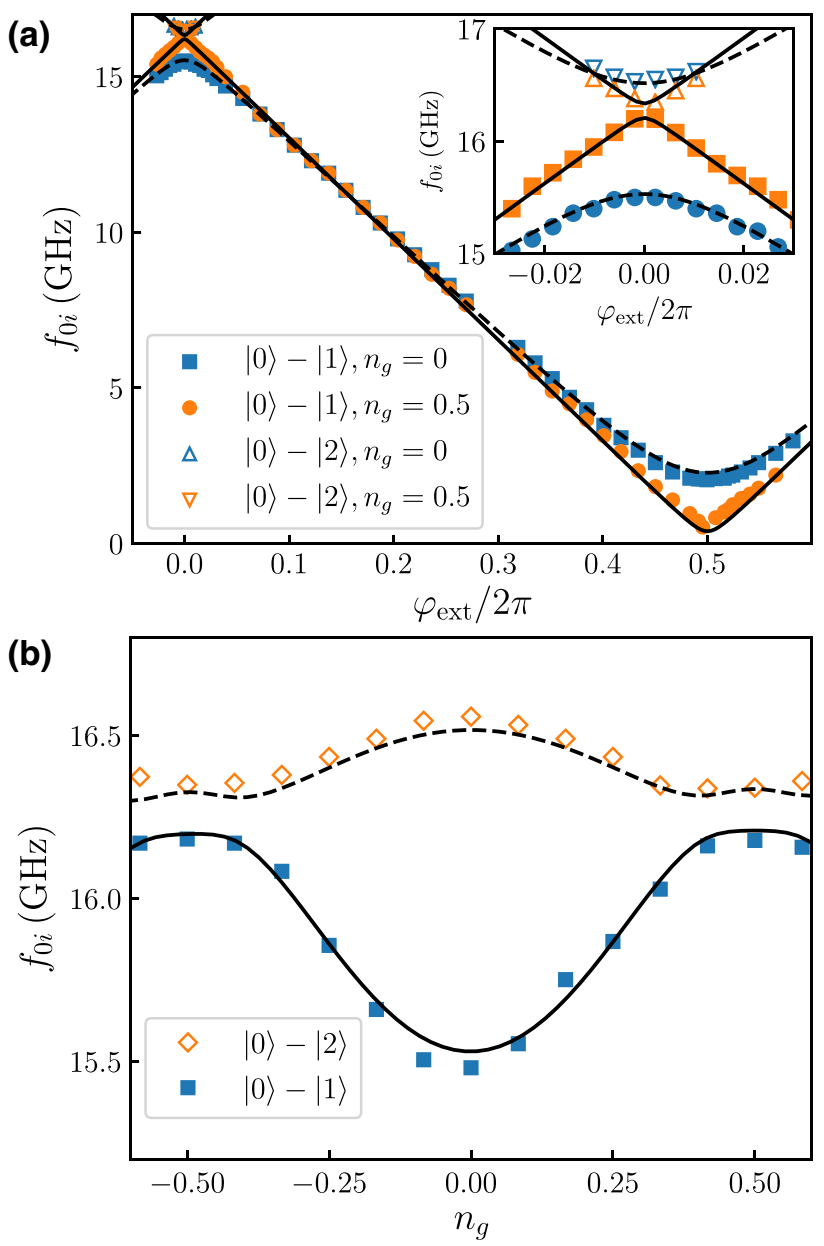

FIG. 4. Spectra of the bifluxon qubit: experimental data for the $|0\rangle-|1\rangle$ and $|0\rangle-|2\rangle$ transitions (symbols) and the result of exact diagonalization of the circuit Hamiltonian in Eq. (1) (solid and dashed lines). (a) Flux dispersion of the transition frequencies $f_{0 i}$ for two values of the CPB charge $n_{g}=0,0.5$. The inset is an enlargement of the qubit spectrum near $\Phi_{\text {ext }}=0$, displaying the avoided crossing that characterizes the rate of double phase slips $E_{\mathrm{dps}}$. (b) Charge dispersion of the $f_{0 i}$ transition frequency for $\phi_{\text {ext }}=0$.

ratio in the spectroscopic measurements is sufficiently high to identify the resonances even in the protected regime. The flux dependencies of the resonance frequencies $f_{01}^{\left(n_{g}\right)}$ and $f_{02}^{\left(n_{g}\right)}$ at $n_{g}=0,0.5$ are shown in Fig. 4(a). The obtained spectra are in good agreement with the results of diagonalization of the circuit Hamiltonian [Eq. (1), solid lines in Fig. 4(a)], with fitting parameters $E_{J} / h=27.2 \mathrm{GHz}, E_{C} / h=7.7 \mathrm{GHz}, E_{L} / h=0.94 \mathrm{GHz}$, and $E_{C L} / h=10 \mathrm{GHz}$, and asymmetry between the CPB junctions $\Delta E_{J}=E_{J 1}-E_{J 2}=h \times 6 \mathrm{GHz}$.

The extracted values are consistent with the expected JJ parameters. The normal-state resistance of the CPB junctions extracted from $E_{J}$ using the Ambegaokar-Baratoff relation agrees within $20 \%$ with the normal-state resistance of test junctions fabricated on the same chip. Both CPB and SI charging energies agree well with the typical aluminum-based junction capacitance $50 \mathrm{fF} / \mu \mathrm{m}^{2}$ and specific capacitance of micron-size islands on silicon substrates $0.085 \mathrm{fF} / \mu \mathrm{m}[29]$.

We also observe an additional resonance at $13.9 \mathrm{GHz}$, whose position does not depend on $\varphi_{\text {ext }}$ and $n_{g}$. We attribute this resonance to the lowest-frequency mode of the superinductor, which corresponds to characteristic impedance of the SI $Z=14 \mathrm{k} \Omega$.

In the time-domain experiments the signal-to-noise ratio, reduced by weak qubit-readout coupling, is too low to employ conventional pulse protocols (decay, Rabi oscillations, and Ramsey fringes). For this reason, we designed special pulse sequences for $T_{1}$ and $T_{2}$ measurements in the protected regime. The pulse sequence used for probing the decay is shown in Fig. 5(a). Initially the qubit is prepared in the ground unprotected state $\left(n_{g}=0\right)$. A microwave $\pi$ pulse at the resonant frequency $f_{01}^{(0)}$ excited the qubit, and then the protection is turned on by applying a pulse of the gate voltage $V_{g}$ corresponding to the offset charge $n_{g}=0.5$. We have used $V_{g}$ pulses with the rise and drop times approximately $30 \mathrm{~ns} \gg 1 /\left(f_{01}^{(0)}-f_{01}^{(0.5)}\right)$,

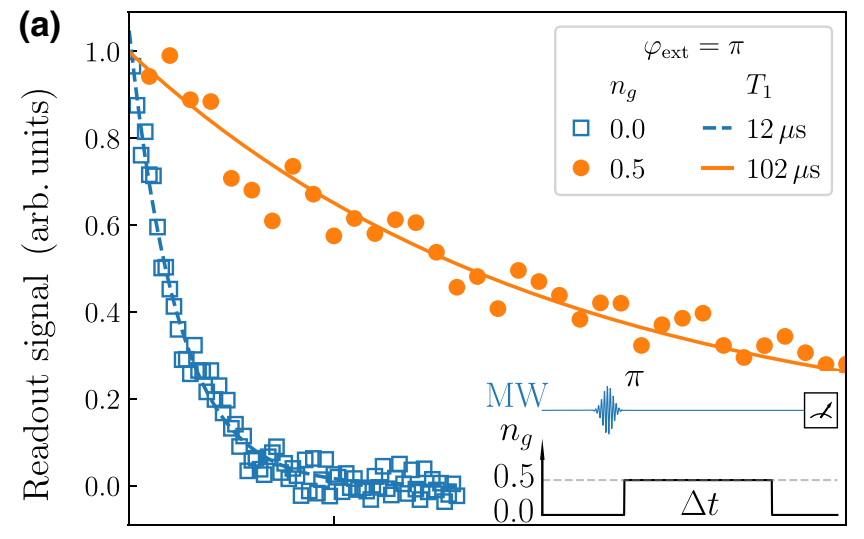

(b)

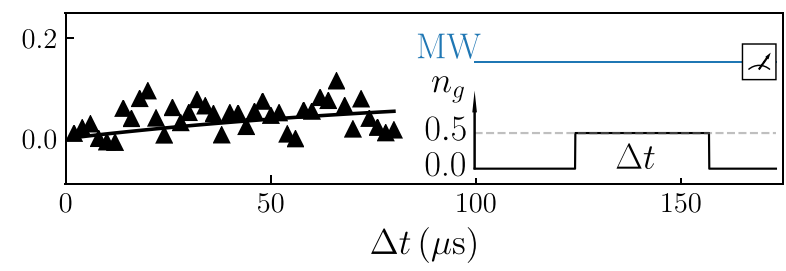

FIG. 5. (a) Measurements of the bifluxon energy relaxation in the protected state (red circles) and unprotected state (blue squares). The sequence of pulses is shown in the inset. The exponential fits are shown by solid and dashed lines, respectively. Note that the resonance energy of the qubit in the protected state is $h \times 0.5 \mathrm{GHz}$ (approximately $k_{B} \times 25 \mathrm{mK}$ ), and a nonzero occupancy of the first excited state $1 /\left[\exp \left(E_{01} / k_{B} T\right)+1\right]$ with the qubit temperature $T \sim 20 \mathrm{mK}$ is taken into account. (b) Demonstration of an absence of qubit excitation by the gate voltage pulses. 
which is sufficiently long to ensure adiabatic evolution of the qubit between protected and unprotected states. After time $\Delta t$, the protection is removed by setting $n_{g}=0$ and the qubit state is measured. As a control experiment, we apply the gate voltage pulses alone, without a $\pi$ pulse; the absence of qubit excitation proved the adiabaticity of gate manipulations; see Fig. 5(b).

The main result of this paper - the dependence of $T_{1}$ on the qubit control parameters $\varphi_{\text {ext }}$ and $n_{g}$-is presented in Fig. 6. Dashed lines represent fits to the model that take into account resistive losses in the capacitively coupled environment and readout resonator (Purcell effect is pronounced near $\varphi_{\text {ext }} / 2 \pi=0.3$ ). The details of the $T_{1}$ calculations are provided in Appendix C. An increase of $T_{1}$ in the protected regime by an order of magnitude provides evidence for the qubit's dipole moment suppression. The longest decay time of greater than $100 \mu \mathrm{s}$ is measured at full flux frustration $\varphi_{\mathrm{ext}}=\pi$, which corresponds to a minimum qubit energy $f_{01}^{(0.5)}=0.4 \mathrm{GHz}$. The routes to further increase $T_{1}$ are discussed in Sec. IV.
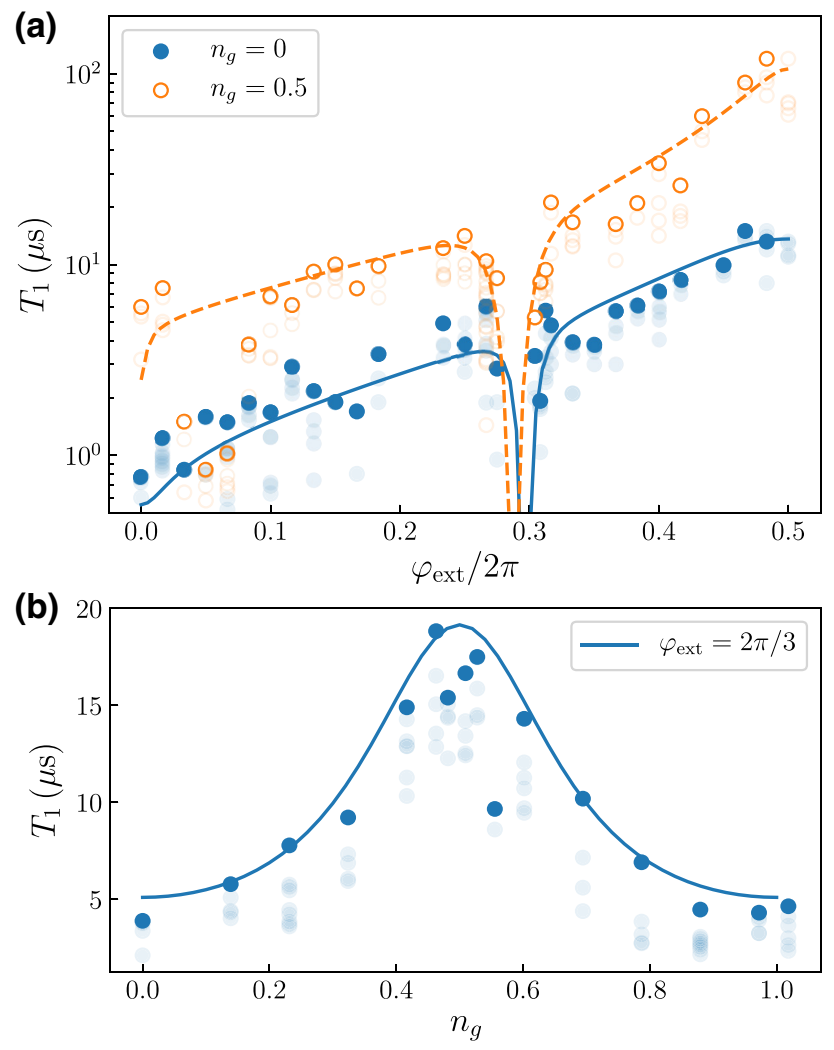

FIG. 6. Energy relaxation time $T_{1}$ as a function of the flux frustration $\phi_{\text {ext }}$ (a) and the CPB charge $n_{g}$ (b). The pale circles represent all the measured data and the bright circles show the longest $T_{1}$ measured for a given operation point. The lines correspond to fitting to the resistive noise theory (Appendix $\mathrm{C}$ ). The sharp dip around $\phi_{\text {ext }}=0.3 \pi$ corresponds to the Purcell decay into the readout resonator.
Direct measurements of the decoherence time $T_{\phi}$ in the protected regime, by either Rabi or Ramsey techniques, are not feasible because of vanishing coupling of the qubit to microwave pulses. For this reason, we modified the measurements of Ramsey fringes by analogy with the aforementioned $T_{1}$ measurements. The pulse sequence is shown in Fig. 7(a). Both $30 \mathrm{~ns}$ long $\pi / 2$ microwave pulses detuned from the qubit transition frequency by $4 \mathrm{MHz}$ are applied in the unprotected state $\left(n_{g}=0\right)$, and the qubit is measured after the end of the second pulse. Between the $\pi / 2$ pulses, while the qubit undergoes free precession, the qubit's protected state is restored by applying a gate voltage pulse $\left(n_{g}=0.5\right)$. After averaging over 1000 cycles, the Ramsey fringes are recorded by varying the delay between the end of the gate pulse and the second $\pi / 2$ pulse.

Ramsey fringes measured according to this procedure for one of the flux "sweet spots" at $\varphi_{\mathrm{ext}}=0$ are shown in Fig. 7(b); the $V_{g}$ pulse for these measurements is $0.27 \mu \mathrm{s}$ long. The difference between the amplitudes of Ramsey fringes at moments $\Delta t=0,0.27 \mu \mathrm{s}$ may provide information on dephasing in the protected state if this is the only source of dephasing. However, the accuracy of this technique is limited by the $V_{g}$ pulse jitter. Indeed, in the rotating frame of the unprotected state, the qubit's state
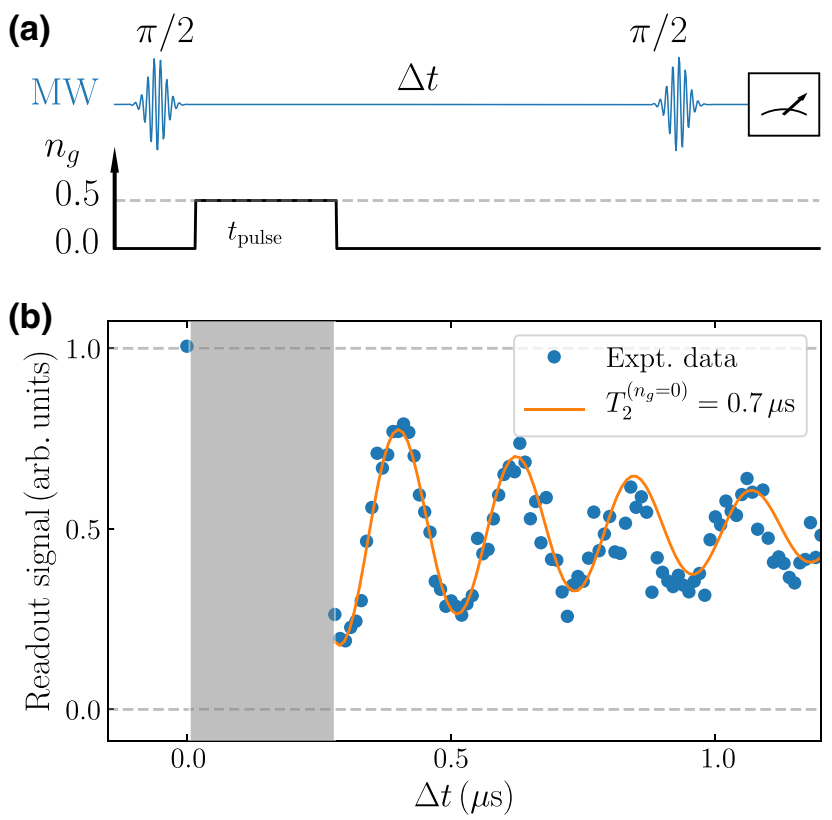

FIG. 7. The Ramsey fringes measurement. (a) The pulse protocol for $T_{2}$ evaluation in the protected state. The protection is turned on for a fixed time of $270 \mathrm{~ns}$; the time delay between two $\pi / 2$ pulses is varied in order to record Ramsey fringes. (b) The experimental data (circles) and the damped-oscillation fitting (the solid line). Note that the value of $T_{2}=0.7 \mu$ s describes the fringe damping in the $n_{g}=0$ state. In the protected state (within a time interval $0<\Delta t<270 \mathrm{~ns}$ ) damping of Ramsey fringes may be caused by the $V_{g}$ pulse jitter rather than dephasing (see the text). 
vector rotates in the equatorial plane of the Bloch sphere as soon as the protection is turned on. The angular velocity of these rotations, $\omega=\left(E_{01}^{(0)}-E_{01}^{(0.5)}\right) / \hbar$, is large $(\omega>$ $2 \pi \times 1 \mathrm{GHz}$ ) at both flux sweet spots $\varphi_{\mathrm{ext}}=0, \pi$; and even a small jitter can result in a significant error in the position of the qubit's state vector at the end of the $V_{g}$ pulse. According to the specification, the jitter time of the pulse generator used in our experiments could be as large as $0.3 \mathrm{~ns}$. This jitter-induced phase uncertainty alone, without invoking any dephasing in the protected state, is sufficient to explain the reduced amplitude of Ramsey fringes at $\Delta t=0.28 \mu \mathrm{s}$. Thus, these measurements can impose only the lower limit on $T_{\phi}$, which is close to $1 \mu \mathrm{s}$ for the data in Fig. 7(b). Future experiments with better controlled $V_{g}$ pulses of different lengths may provide more detailed information on $T_{\phi}$ at both sweet spots.

It should be noted that, since the state of the bifluxon qubit is controlled by the CPB charge $n_{g}$, the device is sensitive to the offset charge drifts and quasiparticle poisoning of the CPB island. In order to eliminate the effect of these fluctuations, $n_{g}$ is measured and, if necessary, readjusted to the desired value before each $T_{1}$ and $T_{2}$ measurement. For calibration, we track one period of the readout dispersive shift oscillations $\delta f_{r}\left(V_{g}\right)$, with minima and maxima corresponding to integer values of the CPB charge. This measurement allows us to estimate the $n_{g}$ drift rate to be less than $10^{-2} / \mathrm{min}$, the quasiparticle tunneling is as rare as 1 event per $30 \mathrm{~min}$ due to the engineered difference between the superconducting gaps in the CPB island and its surroundings (see Appendix E).

\section{DISCUSSION}

In this section we discuss possible modifications to the bifluxon design that could enable further improvement of the qubit coherence beyond the readily available energy decay protection.

First, let us consider the fully symmetric bifluxon qubit with CPB junctions of identical Josephson energy, where charge noise can still potentially flip the fluxon parity and induce energy relaxation. As we have pointed out earlier, the absolute value of the charge dipole moment [Eq. (7)] is strongly suppressed in comparison to that of a conventional charge qubit. Thus, we find that the condition $E_{J} / E_{C L}>10$, similar to the parameter regime of a heavy fluxonium qubit $[5,6]$, is in principle sufficient to achieve $T_{1}$ in excess of $10 \mathrm{~ms}$.

Although the lowest-energy states of the fully symmetric device are exactly degenerate at $\varphi_{\text {ext }} / 2 \pi=n_{g}=0.5$, deviations from this point open a gap in the spectrum, which leads to decoherence [Fig. 8(a)]. A good measure of the qubit sensitivity to pure-dephasing processes is the amplitude of the charge and flux dispersion of the 0-1
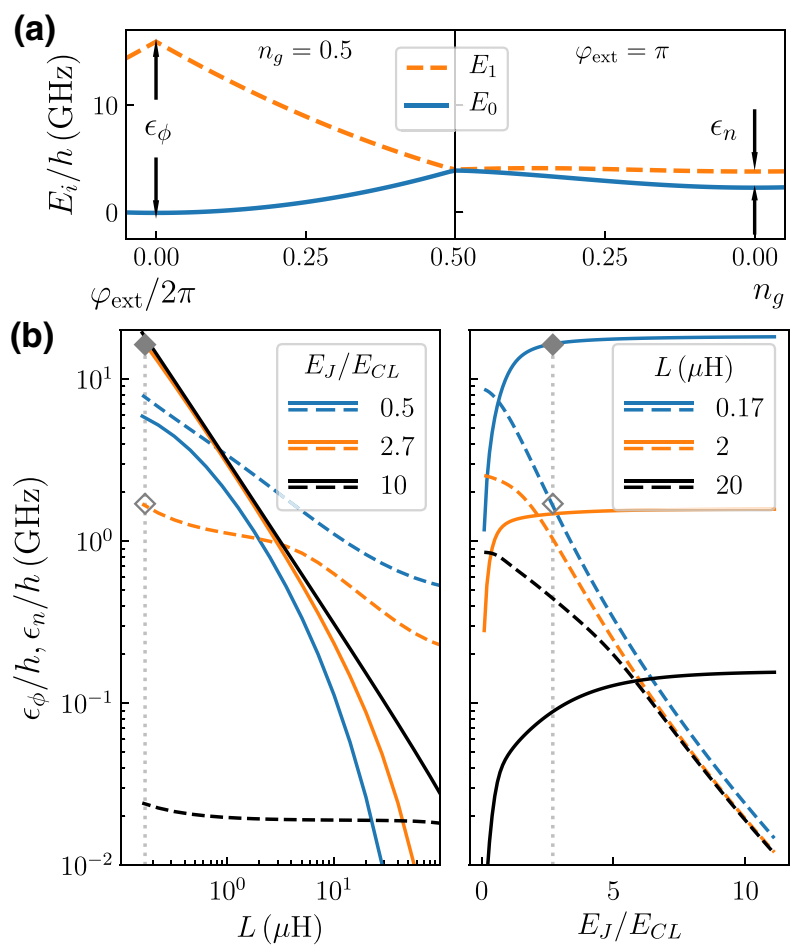

FIG. 8. (a) Two first energy levels of the bifluxon qubit as a function of detuning from degeneracy point. Energy dispersion, which leads to decoherence, can be characterized by amplitudes $\epsilon_{\phi}$ and $\epsilon_{n}$ [see Eq. (10), note that $E_{01}(\pi, 0.5)=0$ for a symmetric device]. (b) Calculated amplitudes of the flux (solid lines) and charge (dashed lines) energy dispersion as a function of qubit parameters. The vertical dotted lines indicate the experimental parameters $L=170 \mathrm{nH}, E_{J} / E_{C L}=2.7$; the filled and open markers show flux and charge dispersion amplitudes of the implemented device, respectively.

transition energy $E_{01}\left(\varphi_{\mathrm{ext}}, n_{g}\right)$, defined as

$$
\begin{aligned}
\epsilon_{\phi} & =E_{01}(0,0.5)-E_{01}(\pi, 0.5), \\
\epsilon_{n} & =E_{01}(\pi, 0)-E_{01}(\pi, 0.5) .
\end{aligned}
$$

As can be seen from Fig. 8(b), in order to mitigate dephasing due to both charge and flux noises, the optimal strategy is to combine an increase of $E_{J} / E_{C L}$ with strong reduction of the inductive energy $E_{L}$. As mentioned above, an exponentially small flux dispersion can be achieved in the regime $E_{\mathrm{dps}} \gg 2 \pi^{2} E_{L}$. Fulfilling this condition requires the implementation of an ultrahigh-impedance superinductor with $L>30 \mu \mathrm{H}$ and self-resonance frequencies greater than $1 \mathrm{GHz}$. Such an element with a characteristic impedance $Z>200 \mathrm{k} \Omega$ could be realized by using strongly disordered superconductor nanowires [30-35] or suspended chains of JJs [28].

If asymmetry between the CPB junctions is present, the SPS amplitude remains nonzero for any charge on the CPB island [Fig. 9(a)]. This leads to mixing of the bifluxon states with different parity and increased susceptibility to 

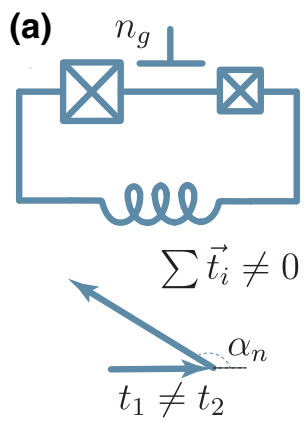

(b)
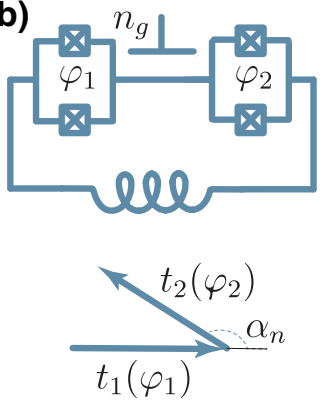

(c)

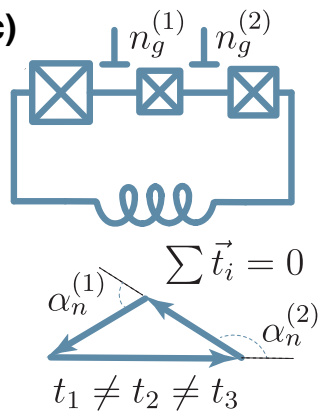

(d)

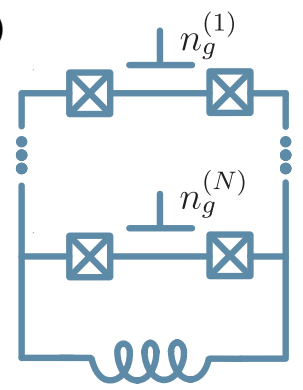

FIG. 9. Possible ways towards further coherence improvements for the bifluxon qubit. (a) Present design: the asymmetry in the JJs of the circuit leads to distinct complex tunneling amplitudes $t_{i}$, represented by the vectors at the bottom. As a result, the single phaseslip rate cannot be completely suppressed for any ac phase $\alpha_{n}=2 \pi n_{g}$. (b) Device with controllable tunneling probability: disorder of the effective Josephson energies can now be mitigated by the local SQUID frustrations $\varphi_{1}$ and $\varphi_{2}$. (c) By adding a second island, the SPS rate can be completely suppressed even for asymmetric junctions as a zero sum of three vectors with comparable lengths. (d) Stacking of the islands into an array: gate charge $n_{g}^{(i)}=0.5$ on any CPB island protects the fluxon parity in the loop. This can be used for expanding the charge sweet spot, similar to Ref. [22].

flux noise. One of the ways to recover the symmetry is to replace the junctions with superconducting quantum interference devices (SQUIDs) of a size much smaller than the bifluxon loop area [see Fig. 9(b)]. This would allow for changing the SQUID's Josephson energy without affecting the optimal flux in the device loop. Alternatively, the SPS can be completely suppressed by introducing a third Josephson junction and an additional gate control line. Indeed, by independently controlling charges on two CPB islands, the SPS amplitude can be tuned to zero [Fig. 9(c)].

The sensitivity of a tunable qubit to fluctuations of a control parameter - the offset charge in our particular case - is the price to pay for the ability to turn on and off the qubit protection and thus facilitate the gate operations. This sensitivity could be suppressed by combining several qubits in a small array [19], as has been demonstrated for the rhombi qubit in Ref. [22]. In a chain of symmetric Josephson rhombi qubits, the transport of single Cooper pairs is forbidden when $\varphi_{\mathrm{ext}}=\pi$ for any rhombus in the chain. Accordingly, the range of values of $\varphi_{\text {ext }}$ where the qubit is protected (i.e., the size of the sweet spot) increases polynomially with the number of rhombi elements in the chain. Similarly, a bifluxon qubit made of a small parallel array of CPBs, as shown in Fig. 9(d), is expected to demonstrate a wider range of $q_{g}$ tunability for which the $|g\rangle$ and $|e\rangle$ states remain degenerate. Realization of such an array would lead to further increase of both the decay and dephasing times beyond the coherence times measured for our proof-of-principle bifluxon-qubit design.

\section{CONCLUSION}

In this work we develop and characterize a quantum superconducting circuit that serves as a platform for the realization of protected qubits with simultaneous exponential suppression of energy decay from charge and flux noise, and dephasing from flux noise. The circuit is realized as a superconducting loop containing a chargesensitive Josephson element (also known as a Cooperpair box) and a superinductor. This circuit with two control parameters - the charge on the $\mathrm{CPB}$ island and the magnetic flux in the loop-is described by a "twodimensional" Hamiltonian. Its dimensionality $D>1$ is critical to simultaneous suppression of decay and dephasing via localization of the qubit's wavefunctions in disparate regions of the phase space. The ability to turn the protection on and off by controlling the charge on the CPB island facilitates gate operations with protected qubits. By switching the protection on, we observe a tenfold increase of the decay time, up to $100 \mu \mathrm{s}$. The studied circuit is not expected to demonstrate a long dephasing time because of its sensitivity to fluctuations of charge on the CPB island. However, the bifluxon sensitivity to charge noise is much reduced in comparison with the charge qubit, and the charge-noise-induced dephasing time in the protected state exceeded $1 \mu \mathrm{s}$. Further improvement of the coherence times can be achieved in the next-generation devices by the optimization of their parameters and combining several $\cos (\phi / 2)$ elements in a small array.

\section{ACKNOWLEDGMENTS}

We would like to thank Elio König, Yashar Komijani, Lev Ioffe, and Vladimir Manucharyan for helpful discussions. The work at Rutgers University was supported in part by NSF Grants No. DMR-1708954 and No. DMR1838979, and ARO Grant No. W911NF-17-C-0024. The work at the University of Massachusetts Boston was supported in part by a 2019 Google Faculty Research Grant and NSF Grants No. ECCS-1608448, No. DUE-1723511, and No. DMR-1838979. The work at the Université of 
Sherbrooke was supported in part by the NSERC and the Canada First Research Excellence Fund.

\section{APPENDIX A: DERIVATION OF THE CIRCUIT HAMILTONIAN}

In this section, we derive the full device Hamiltonian, including the bifluxon qubit, the readout resonator, and the coupling between these circuits.

A lumped-element circuit model for the device is presented in Fig. 10. The readout resonator (with inductance $L_{R}$, capacitance $C_{R}$ and capacitance to the ground $\left.C_{\text {gnd }}\right)$ is coupled to the bifluxon circuit through the shared inductance $L_{\mathrm{sh}}$. Following the standard circuit-quantization procedure [36], we arrive at a circuit Lagrangian of the form

$$
\begin{aligned}
\mathcal{L}= & \frac{C_{0}}{2}\left(\dot{\Phi}_{a}^{2}+\dot{\Phi}_{b}^{2}\right)+\frac{C_{0_{g}}}{2} \dot{\Phi}^{2}+\frac{C_{g}}{2}\left(\dot{\Phi}-V_{g}\right)^{2} \\
& +\frac{C_{J}}{2}\left[\left(\dot{\Phi}-\dot{\Phi}_{a}\right)^{2}+\left(\dot{\Phi}-\dot{\Phi}_{b}\right)^{2}\right] \\
& +E_{J} \cos \left[\frac{2 \pi}{\Phi_{0}}\left(\Phi-\Phi_{a}+\frac{\Phi_{\mathrm{ext}}}{2}\right)\right] \\
& +E_{J} \cos \left[\frac{2 \pi}{\Phi_{0}}\left(\Phi-\Phi_{b}-\frac{\Phi_{\mathrm{ext}}}{2}\right)\right] \\
& -\frac{1}{2 L / 2}\left[\left(\Phi_{a^{\prime}}-\Phi_{a}\right)^{2}+\left(\Phi_{b}-\Phi_{b^{\prime}}\right)^{2}\right] \\
& +\frac{C_{a^{\prime}}}{2} \dot{\Phi}_{a^{\prime}}^{2}+\frac{C_{b^{\prime}}}{2} \dot{\Phi}_{b^{\prime}}^{2}-\frac{\left(\Phi_{b^{\prime}}-\Phi_{a^{\prime}}\right)^{2}}{2 L_{\mathrm{sh}}} \\
& +\frac{C_{R}}{2}\left(\dot{\Phi}_{r}-\dot{\Phi}_{a^{\prime}}\right)^{2}-\frac{\left(\Phi_{r}-\Phi_{b^{\prime}}\right)^{2}}{2 L_{R}}
\end{aligned}
$$

where we have defined the flux node variables $\left\{\Phi, \Phi_{a}, \Phi_{b}\right.$, $\left.\Phi_{a^{\prime}}, \Phi_{b^{\prime}}, \Phi_{r}\right\}$ corresponding to the degrees of freedom of

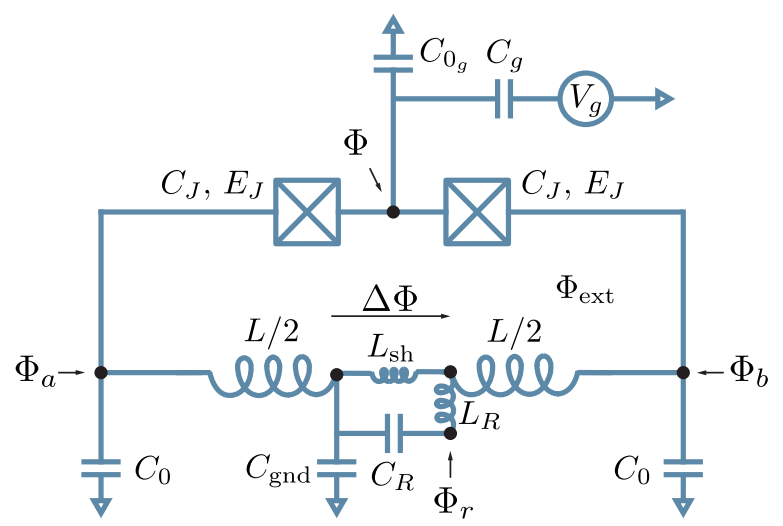

FIG. 10. Schematic diagram of the bifluxon device including the readout resonator. The scheme also includes gate, ground, and stray capacitances of the circuit. Moreover, $V_{g}$ denotes the gate voltage that sets the offset charge $n_{g}^{\varphi}$. the circuit in Fig. 10, the gate voltage $V_{g}$, and the external magnetic flux $\Phi_{\text {ext }}$. Moreover, $C_{a^{\prime}}$ and $C_{b^{\prime}}$ denote effective capacitances for nodes $a^{\prime}$ and $b^{\prime}$ of the circuit, respectively.

To obtain a simpler model that still adequately describes the experiment, we eliminate the passive circuit modes $\Phi_{a^{\prime}}$ and $\Phi_{b^{\prime}}$. We do this by assuming that the flux difference $\Phi_{-}=\Phi_{b}-\Phi_{a}$ builds up linearly between nodes $a$ and $b$ (see Fig. 10). This assumption is represented by the relations

$$
\begin{aligned}
& \Phi_{a^{\prime}} \simeq \frac{L / 2}{L+L_{\mathrm{sh}}} \Phi_{-}+\Phi_{a}, \\
& \Phi_{b^{\prime}} \simeq-\frac{L / 2}{L+L_{\mathrm{sh}}} \Phi_{-}+\Phi_{b},
\end{aligned}
$$

which allow us to approximate the inductive energy of the bifluxon circuit as

$$
\frac{1}{2 L / 2}\left[\left(\Phi_{a^{\prime}}-\Phi_{a}\right)^{2}+\left(\Phi_{b}-\Phi_{b^{\prime}}\right)^{2}\right]+\frac{\left(\Phi_{b^{\prime}}-\Phi_{a^{\prime}}\right)^{2}}{2 L_{\mathrm{sh}}} \simeq \frac{\Phi_{-}^{2}}{2 L}
$$

Note that the latter expression disregards a leading-order correction of the form $2\left(L_{\mathrm{sh}} / L\right) \Phi_{-}^{2} / 2 L$, which is assumed to be negligible in the weak-coupling regime $L_{\mathrm{sh}} / L \ll 1$. Additionally, we assume that the capacitances $C_{a^{\prime}}$ and $C_{b^{\prime}}$, which are unknown in the experiment, contribute very little to the total mass of modes $\Phi_{a}, \Phi_{b}$, and $\Phi_{-}$. In this case we can approximate Eq. (A1) as

$$
\begin{aligned}
\mathcal{L}= & \frac{C_{0}}{2}\left(\dot{\Phi}_{a}^{2}+\dot{\Phi}_{b}^{2}\right)+\frac{C_{0_{g}}}{2} \dot{\Phi}^{2}+\frac{C_{g}}{2}\left(\dot{\Phi}-V_{g}\right)^{2} \\
& +\frac{C_{J}}{2}\left[\left(\dot{\Phi}-\dot{\Phi}_{a}\right)^{2}+\left(\dot{\Phi}-\dot{\Phi}_{b}\right)^{2}\right]-\frac{1}{2 L} \Phi_{-}^{2} \\
& +E_{J} \cos \left[\frac{2 \pi}{\Phi_{0}}\left(\Phi-\Phi_{a}+\frac{\Phi_{\mathrm{ext}}}{2}\right)\right] \\
& +E_{J} \cos \left[\frac{2 \pi}{\Phi_{0}}\left(\Phi-\Phi_{b}-\frac{\Phi_{\mathrm{ext}}}{2}\right)\right] \\
& +\frac{C_{R}}{2}\left(\dot{\Phi}_{r}-\dot{\Phi}_{a^{\prime}}\right)^{2}-\frac{\left(\Phi_{r}-\Phi_{b^{\prime}}\right)^{2}}{2 L_{R}} .
\end{aligned}
$$

The fifth line of Eq. (A4), where $\Phi_{a^{\prime}}$ and $\Phi_{b^{\prime}}$ are given in Eq. (A2), includes both capacitive and inductive coupling of the bifluxon variables to the readout resonator. While taking into account these two coupling mechanisms is not particularly difficult from a theoretical point of view, here we are interested in describing the effective inductive coupling that dominates in the experiment. For the sake of simplicity, we thus neglect the capacitive coupling term, rewrite $\Phi_{b^{\prime}}=\left[L_{\mathrm{sh}} / 2\left(L+L_{\mathrm{sh}}\right)\right] \Phi_{-}+\Phi_{+} / 2$, where 
$\Phi_{+}=\Phi_{b}+\Phi_{a}$, and expand the inductive coupling as

$$
\frac{\left(\Phi_{r}-\Phi_{b^{\prime}}\right)^{2}}{2 L_{R}}=\frac{\Phi_{r}^{2}}{2 L_{R}}-\frac{1}{2\left(L+L_{\mathrm{sh}}\right)} \eta_{\mathrm{sh}} \Phi_{r} \Phi_{-}+\cdots,
$$

where the ellipses represent interaction terms that involve the $\Phi_{+}$mode (excluded below) and $\eta_{\mathrm{sh}}=L_{\mathrm{sh}} / L_{R}$ is an inductive participation ratio.

Performing the transformations $\Phi_{a} \rightarrow \Phi_{a}+\Phi_{\text {ext }} / 2$ and $\Phi_{b} \rightarrow \Phi_{b}-\Phi_{\text {ext }} / 2$, the simplified circuit Lagrangian reduces to

$$
\begin{aligned}
\mathcal{L}= & \frac{C_{0_{c}}+C_{g}+2 C_{J}}{2} \dot{\Phi}^{2}+\frac{C_{0}+C_{J}}{4}\left(\dot{\Phi}_{-}^{2}+\dot{\Phi}_{+}^{2}\right) \\
& -C_{J} \dot{\Phi} \dot{\Phi}_{+}-C_{g} \dot{\Phi} V_{g}-\frac{1}{2 L}\left(\Phi_{-}-\Phi_{\mathrm{ext}}\right)^{2} \\
& +2 E_{J} \cos \left[\frac{2 \pi}{\Phi_{0}} \frac{\Phi_{-}}{2}\right] \cos \left[\frac{2 \pi}{\Phi_{0}}\left(\Phi-\frac{\Phi_{+}}{2}\right)\right] \\
& +\frac{C_{R}}{2} \dot{\Phi}_{r}^{2}-\frac{\Phi_{r}^{2}}{2 L_{R}}+\frac{1}{2\left(L+L_{\mathrm{sh}}\right)} \eta_{\mathrm{sh}} \Phi_{r} \Phi_{-}+\cdots,
\end{aligned}
$$

and by means of a Legendre transformation we arrive at the effective circuit Hamiltonian

$$
\begin{aligned}
H= & 4 E_{C_{\varphi}}\left(n_{\varphi}-n_{g}^{\varphi}\right)^{2}+4 E_{C_{\phi}} n_{\phi}^{2}+4 E_{C_{\phi_{+}}} n_{\phi_{+}}^{2} \\
& -2 E_{J} \cos \left(\frac{\phi}{2}\right) \cos \left(\varphi-\frac{\phi_{+}}{2}\right)+\frac{E_{L}}{2}\left(\phi-\varphi_{\mathrm{ext}}\right)^{2} \\
& +\hbar g_{\varphi \phi_{+}} n_{\varphi} n_{\phi_{+}}+\hbar \omega_{R} a^{\dagger} a+\frac{\eta_{\mathrm{sh}}}{2} E_{L} \phi_{r} \phi+\cdots .
\end{aligned}
$$

Here, we have defined the phase variables $\varphi=\Phi / \varphi_{0}$, $\phi=\Phi_{-} / \varphi_{0}$, and $\phi_{+}=\Phi_{+} / \varphi_{0}$, the respective conjugate charge operators $n_{\varphi}, n_{\phi}$, and $n_{\phi_{+}}$, and the charging energies $E_{C_{\mu}}=e^{2} / 2 C_{\mu}$ for $\mu \in\left[\varphi, \phi, \phi_{+}\right]$in terms of the mode capacitances

$$
\begin{gathered}
C_{\varphi}=C^{2} /\left(C_{0}+C_{J}\right), \\
C_{\phi}=\left(C_{0}+C_{J}\right) / 2, \\
C_{\phi_{+}}=C^{2} / 2\left(C_{0_{g}}+C_{g}+2 C_{J}\right),
\end{gathered}
$$

with $C^{2}=\left(C_{0_{g}}+C_{g}\right) C_{J}+C_{0}\left(C_{0_{g}}+C_{g}+2 C_{J}\right)$. We also introduce an effective coupling constant $\hbar g_{\varphi \phi_{+}}=e^{2} / 2$ $C_{\varphi \phi_{+}}$, where $C_{\varphi \phi_{+}}=C^{2} /\left(16 C_{J}\right)$, and the offset charge $n_{g}^{\varphi}=-\beta_{\varphi}\left(2 e V_{g} / 8 E_{C_{\varphi}}\right)$ with $\beta_{\varphi}=C_{g} / C_{\varphi}$. The resonator Hamiltonian is written in terms of its resonance frequency
$\omega_{R}$ and the ladder operators $\left(a, a^{\dagger}\right)$, and we have approximated $\left(\Phi_{0} / 2 \pi\right)^{2} /\left(L+L_{\mathrm{sh}}\right) \simeq\left(\Phi_{0} / 2 \pi\right)^{2} / L=E_{L}$.

We now assume that $\phi_{+}$is a high-frequency mode detuned away from the qubit transitions of interest [37]. Under this approximation, the coupling $g_{\varphi \phi_{+}}$leads to a small dispersive shift that is not required to describe the experimental data of the device studied in this work. Thus, by disregarding the $\phi_{+}$mode, Eq. (A7) reduces to a model of a two-dimensional $(\varphi, \phi)$ qubit Hamiltonian coupled to the resonator mode $\phi_{r}$, which we use in the main text. Following this approximation, we also exclude the terms of the Hamiltonian Eq. (A7) that are represented by ellipses, as these terms describe spurious coupling of the $\phi_{+}$mode to $\phi_{-}$and $\phi_{r}$. Finally, we note that, for simplicity, the capacitive energies $E_{C_{\varphi}}$ and $E_{C_{\phi}}$ in Eq. (A7) are respectively denoted by $E_{C_{\varphi}} \rightarrow E_{C}$ and $E_{C_{\phi}} \rightarrow E_{C L}$ in the main text and in the appendices below.

Finally, in order to account for the effect of circuitelement disorder on the circuit junctions, we derive a perturbative correction to Eq. (A7) of the form

$$
\delta H=\Delta E_{J} \sin (\phi / 2) \sin \left(\varphi-\phi_{+} / 2\right),
$$

where $\Delta E_{J}=E_{J a}-E_{J b}$ is the junction asymmetry, defined in terms of the junction energies $E_{J a}$ and $E_{J b}$, and $\bar{E}_{J}=\left(E_{J a}+E_{J b}\right) / 2$. Note that the replacement $E_{J} \rightarrow \bar{E}_{J}$ in Eq. (A7) should also be made.

\section{APPENDIX B: INTEGER CHARGE ON THE ISLAND $n_{g}=N$}

Let us consider the reduced Hamiltonian Eq. (2) for the case of an integer charge $n_{g}$ on the CPB island. In the limit $E_{C} \gg E_{J}$, we can restrict the analysis to two charge states. The matrix representation of the Hamiltonian then reads

$$
H=\left(\begin{array}{cc}
\mathbb{A}+\mathbb{C} & -\mathbb{B} \\
-\mathbb{B} & \mathbb{A}-\mathbb{C}
\end{array}\right),
$$

where

$$
\begin{aligned}
& \mathbb{A}=-4 E_{C L} \partial_{\phi}^{2}+\frac{E_{L}}{2}\left(\phi-\varphi_{\mathrm{ext}}\right)^{2}, \\
& \mathbb{B}=E_{J} \cos (\phi / 2), \quad \mathbb{C}=2 E_{C} .
\end{aligned}
$$

If $\mathbb{C}$ is the dominant term in Eq. (B1), the charge component of the lowest energy eigenvector is close to a pure $|N\rangle$ state

$$
\left|\psi^{n}\right\rangle=\left(\begin{array}{c}
\alpha \\
1
\end{array}\right)
$$

for $\alpha \ll 1$. The eigenvalue

$$
\mathbb{E}=\mathbb{A}-\sqrt{\mathbb{C}^{2}+\mathbb{B}^{2}} \approx \mathbb{A}-\mathbb{C}-\frac{\mathbb{B}^{2}}{2 \mathbb{C}}
$$


corresponds to a fluxonium-like Hamiltonian of the form

$$
H=-4 E_{C L} \partial_{\phi}^{2}+\frac{1}{2} E_{L}\left(\phi-\varphi_{\mathrm{ext}}\right)^{2}-E_{J}^{*} \cos \phi,
$$

where $E_{J}^{*}=E_{J}^{2} / 4 E_{C}$ is a renormalized Josephson energy.

\section{APPENDIX C: COUPLING TO THE ENVIRONMENT AND DECOHERENCE}

In this section, we consider the coupling of the qubit modes to environmental sources of noise, and derive the relaxation rates that are used in the main text to fit $T_{1}$. In Fig. 11 we illustrate the coupling of the bifluxon qubit to external (noisy) degrees of freedom.

Charge-induced decay occurs due to coupling of the CPB phase $\varphi$ to the environment, mainly via the voltage line that is used to control $n_{g}^{\varphi}$. The coupling Hamiltonian is of the form $\delta H=2 e n_{\varphi} \beta_{\varphi} \Delta V$, where $\Delta V$ is a voltagenoise operator leading to fluctuations of the offset charge. Using Fermi's golden rule, we derive the transition rate $\Gamma_{1 \rightarrow 0}=\left|\left\langle 0\left|2 e n_{\varphi} \beta_{\varphi}\right| 1\right\rangle\right|^{2} S_{V}\left(\omega_{01}\right) / \hbar^{2}$, where $S_{V}\left(\omega_{01}\right)$ is the noise spectral density evaluated at the qubit transition frequency. Denoting the impedance of the environment coupled to the qubit port as $Z(\omega)$ and assuming an Ohmic spectral density of the form $S_{V}(\omega)=\hbar \omega \operatorname{Re}[Z(\omega)][1+$ $\operatorname{coth}\left(\hbar \omega / 2 k_{B} T\right)$ ] [36], we arrive at the expression [38,39]

$$
\Gamma_{1 \rightarrow 0}^{(n)}=\beta_{\varphi}^{2}\left|\left\langle 0\left|n_{\varphi}\right| 1\right\rangle\right|^{2} r_{\mathrm{env}} \omega_{01}\left[1+\operatorname{coth}\left(\frac{\hbar \omega_{01}}{2 k_{B} T}\right)\right],
$$

where $r_{\text {env }}=\operatorname{Re}\left[Z\left(\omega_{01}\right)\right] / R_{K}$ is the effective resistance of the environment in units of the reduced superconducting quantum of resistance, $R_{K}=\hbar /(2 e)^{2} \simeq 1 \mathrm{k} \Omega$.

Coupling of noise to the fluxonium-like degree of freedom $(\phi)$ can be treated similarly. Instead of rewriting Eq. (C1) for $n_{\phi}$, however, we derive an expression that involves the transition matrix elements of the phase operator. As a consequence of the commutation relation $\left[\phi, n_{\phi}\right]=i,[\phi, H]=i 8 E_{C L} n_{\phi}$ and thus $\hbar \omega_{01}\langle 0|\phi| 1\rangle=$

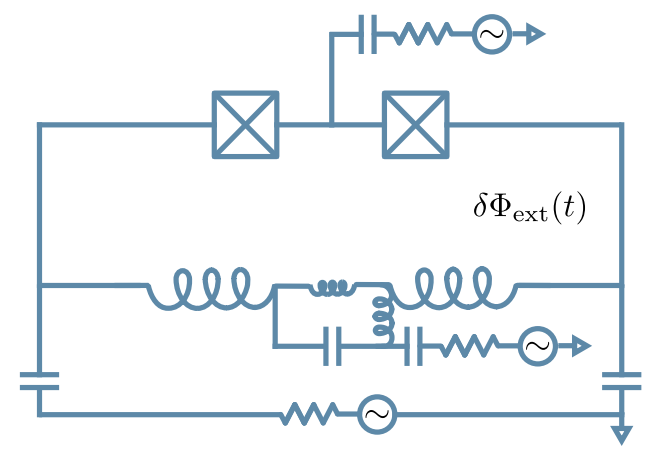

FIG. 11. Bifluxon device coupled to environmental degrees of freedom leading to decoherence. The resistors model dissipative circuit elements coupled capacitively to the qubit. $\delta \Phi_{\text {ext }}(t)$ represents the magnetic flux fluctuations. $i 8 E_{C L}\left\langle 0\left|n_{\phi}\right| 1\right\rangle$ [40], we can rewrite Eq. (C1) as

$$
\Gamma_{1 \rightarrow 0}^{(\phi)}=\beta_{\phi}^{2}|\langle 0|\phi| 1\rangle|^{2} r_{\mathrm{env}}\left(\frac{\hbar \omega_{01}}{8 E_{C L}}\right)^{2} \omega_{01}\left[1+\operatorname{coth}\left(\frac{\hbar \omega_{01}}{2 k_{B} T}\right)\right]
$$

While expressions similar to Eqs. (C1) and (C2) can be derived for the excitation rates $\Gamma_{0 \rightarrow 1}^{(n)}$ and $\Gamma_{0 \rightarrow 1}^{(\phi)}$, respectively, these rates are negligible compared to the relaxation rates at the qubit operation frequencies. For this reason, $T_{1}^{\mathrm{nc}}=1 / \Gamma_{1 \rightarrow 0}^{\mathrm{nc}}+1 / \Gamma_{0 \rightarrow 1}^{\mathrm{nc}}$, where the superscript "nc" denotes a generic noise channel, can be safely approximated by $T_{1}^{\mathrm{nc}} \simeq 1 / \Gamma_{1 \rightarrow 0}^{\mathrm{nc}}$.

Finally, we discuss Purcell decay due to coupling of the qubit to the readout resonator. By rewriting the qubit-resonator coupling in Eq. (A7) as $\delta H=$ $\eta_{\mathrm{sh}} E_{L} \phi \sqrt{z_{r} / 2}\left(a+a^{\dagger}\right) / 2$, where $z_{r}=Z_{r} / R_{K}$ is the reduced resonator impedance and following Ref. [17], we arrive at

$$
\Gamma_{1 \rightarrow 0}^{(\mathrm{P})}=\eta_{\mathrm{sh}}^{2} \frac{z_{r}}{8} \frac{\omega_{r}}{Q_{r}} \frac{\left(E_{L} / \hbar\right)^{2}}{\left|\omega_{01}-\omega_{r}\right|^{2}},
$$

where $Q_{r}$ is the quality factor of the readout resonator. The Purcell rate makes a significant contribution to $T_{1}$ only close to the readout resonance frequency, while away from this narrow range the qubit relaxation time is well described by the sum of the two terms given in Eqs. (C1) and $(\mathrm{C} 2)$.

In order to fit $T_{1}$, we use only two fitting parameters, $\kappa_{\phi}=\beta_{\phi}^{2} r_{\text {env, } \phi}$ and $\kappa_{\varphi}=\beta_{\varphi}^{2} r_{\text {env }, \varphi}$. As can be seen from Fig. 3(c), the former determines $T_{1}$ at $n_{g}=0$ and the latter at $n_{g}=0.5$. We require the parameters $\beta$ and $r_{\text {env }}$ (and thereby $\kappa_{\phi, \varphi}$ ) to be small compared to unity. More accurate separation of the coupling and noise amplitude contributions is not feasible at this moment due to a lack of knowledge of the full capacitance matrix of the device.

\section{APPENDIX D: SAMPLE PARAMETERS}

In Tables I-III we present summaries of the sample parameters.

TABLE I. The bifluxon qubit parameters estimated for a test structure: Josephson and charging energies, areas of the junctions in the CPB and SI array, and the number of junctions in the array and its total inductance.

\begin{tabular}{lccccc}
\hline \hline & $\begin{array}{c}E_{J} / h \\
(\mathrm{GHz})\end{array}$ & $\begin{array}{c}E_{C J} / h \\
(\mathrm{GHz})\end{array}$ & $\begin{array}{c}S_{J} \\
\left(\mathrm{~nm}^{2}\right)\end{array}$ & $N_{A}$ & $\begin{array}{c}L \\
(\mathrm{nH})\end{array}$ \\
\hline SI & 114.7 & 6.1 & $210 \times 300$ & 122 & 170 \\
$\mathrm{CPB}$ & 32.0 & 21.9 & $110 \times 160$ & & \\
\hline \hline
\end{tabular}


TABLE II. The resonator parameters: inductance, capacitance, shunting inductance, and loaded and intrinsic quality factors.

\begin{tabular}{lccccc}
\hline \hline $\begin{array}{l}L_{R} \\
(\mathrm{nH})\end{array}$ & $\begin{array}{c}C_{R} \\
(\mathrm{fF})\end{array}$ & $\begin{array}{c}L_{\mathrm{sh}} \\
(\mathrm{nH})\end{array}$ & $\begin{array}{c}f_{r} \\
(\mathrm{GHz})\end{array}$ & $Q_{l}$ & $Q_{\text {int }}$ \\
\hline 4 & 120 & 0.4 & 6.5 & $5 \times 10^{3}$ & $50 \times 10^{3}$ \\
\hline \hline
\end{tabular}

\section{APPENDIX E: GAP ENGINEERING FOR MITIGATION OF QUASIPARTICLE POISONING}

Quasiparticle poisoning (QP) presents a problem for charge-sensitive quantum superconducting devices [41, 42]. In particular, for a bifluxon qubit in a protected state, tunneling of a nonequilibrium quasiparticle into or out of the CPB island would remove protection. To minimize QP, we use so-called gap engineering [43,44]. In Fig. 12(a) we show the superconducting gap in the $\mathrm{CPB}$ island and the outer electrodes that form the CPB Josephson junctions. Because of the dependence of the critical temperature of Al films on their thickness, the gap in the thin $(20 \mathrm{~nm})$ CPB island is greater than that in thicker $(60 \mathrm{~nm})$ outer electrodes. This difference $\delta \Delta$, which we estimate to be approximately $(0.3-0.4) \mathrm{K}$ (see, e.g., [46]), is sufficiently large to block tunneling of nonequilibrium quasiparticles with energies greater than $\delta \Delta$ onto the $\mathrm{CPB}$ island at sufficiently low temperatures.

The efficiency of this technique is demonstrated in Figs. 1(b) $-1(d)$. If both the CPB island and outer electrodes are thick $(\delta \Delta \simeq 0)$, we observe a characteristic "eye" pattern [43] in the spectroscopic measurements, which reflects rapid $\pm e$ jumps of the $\mathrm{CPB}$ charge on the timescale of a single scan of the resonance of the readout resonator; see Fig. 12(b). This pattern vanishes if the gap engineering is employed and reappears only at higher temperatures, where the quasiparticles are thermally excited in the CPB island [compare panels (c) and (d) of Fig. 12]. Gap engineering and careful infrared and magnetic shielding of the device allow us to increase the time intervals between the QP events up to 30 mins. In Fig. 12(e) we show that, in addition to rare QP events, in the gap-engineered device we observe slow monotonic drift of $n_{g}$ whose origin remains unclear. Because of this drift, we have to measure (and, if necessary, readjust) $n_{g}$ before each time-domain measurement.

TABLE III. The fitting parameters: the CPB junction Josephson energy, charging energies of $\mathrm{CPB}$ and SI, the SI inductive energy, the CPB junctions asymmetry, and noise factors of SI and CPB modes.

\begin{tabular}{lcccccc}
\hline \hline $\begin{array}{l}E_{J} / h \\
(\mathrm{GHz})\end{array}$ & $\begin{array}{c}E_{C} / h \\
(\mathrm{GHz})\end{array}$ & $\begin{array}{c}E_{C L} / h \\
(\mathrm{GHz})\end{array}$ & $\begin{array}{c}E_{L} / h \\
(\mathrm{GHz})\end{array}$ & $\begin{array}{c}\Delta E_{J} / h \\
(\mathrm{GHz})\end{array}$ & $\begin{array}{c}\kappa_{\phi} \\
\times 10^{-3}\end{array}$ & $\begin{array}{c}\kappa_{\varphi} \\
\times 10^{-3}\end{array}$ \\
\hline 27.2 & 7.7 & 10.0 & 0.94 & 6 & 0.7 & 2 \\
\hline \hline
\end{tabular}

(a)
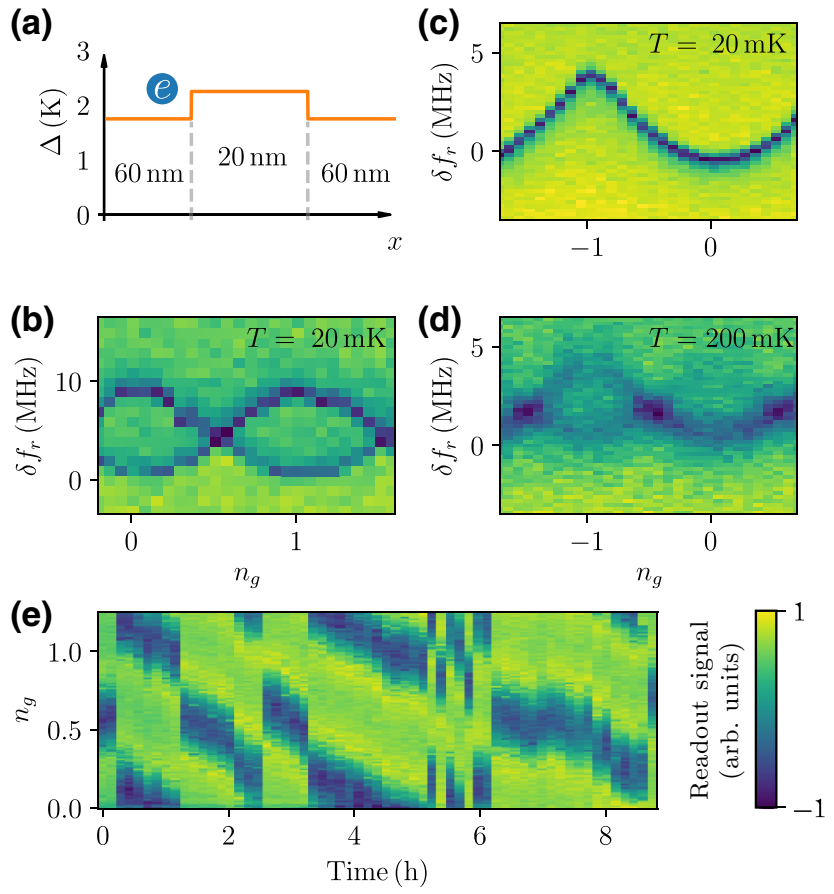

FIG. 12. Suppression of quasiparticle poisoning by gap engineering. (a) Profile of the superconducting gap across the CPB island. The critical temperature of the thin $\mathrm{CPB}$ island is $0.2-0.3 \mathrm{~K}$ higher than that in the thicker electrodes. (b)-(d) Spectroscopy of the readout resonator as a function of $q_{g}$ for bifluxon qubits: without gap modulation at $20 \mathrm{mK}$ (b), and with gap modulation at $20 \mathrm{mK}$ (c) and $200 \mathrm{mK}$ (d). (e) The gap-engineered device at $20 \mathrm{mK}$. The dispersive shift $\delta f_{r}$ of the readout resonator (color coded) is measured at a fixed gate voltage $V_{g}$ over $9 \mathrm{~h}$. The shift $\delta f_{r}$ is converted into $\delta n_{g}$ using the data of panel (c). Abrupt jumps reflect the QP events $\left(\delta n_{g}= \pm 0.5\right)$, gradual shift corresponds to a monotonic drift of $n_{g}$ with a rate of less than $10^{-2} / \mathrm{min}$.

[1] M. Kjaergaard, M. E. Schwartz, J. Braumüller, P. Krantz, J. I.-J. Wang, S. Gustavsson, and W. D. Oliver, Superconducting qubits: Current state of play, arXiv:1905.13641 (2019).

[2] M. H. Devoret and R. J. Schoelkopf, Superconducting circuits for quantum information: An outlook, Science 339, 1169 (2013).

[3] J. Preskill, Quantum computing in the NISQ era and beyond, Quantum 2, 79 (2018).

[4] C. Müller, J. H. Cole, and J. Lisenfeld, Towards understanding two-level-systems in amorphous solids - insights from quantum devices, arXiv:1705.01108 (2017).

[5] N. Earnest, S. Chakram, Y. Lu, N. Irons, R. K. Naik, N. Leung, L. Ocola, D. A. Czaplewski, B. Baker, J. Lawrence, J. Koch, and D. I. Schuster, Realization of a $\lambda$ System with Metastable States of a Capacitively Shunted Fluxonium, Phys. Rev. Lett. 120, 150504 (2018).

[6] Y.-H. Lin, L. B. Nguyen, N. Grabon, J. San Miguel, N. Pankratova, and V. E. Manucharyan, Demonstration of 
Protection of a Superconducting Qubit from Energy Decay, Phys. Rev. Lett. 120, 150503 (2018).

[7] D. Vion, A. Aassime, A. Cottet, P. Joyez, H. Pothier, C. Urbina, D. Esteve, and M. H. Devoret, Manipulating the quantum state of an electrical circuit, Science 296, 886 (2002).

[8] A. A. Houck, J. Koch, M. H. Devoret, S. M. Girvin, and R. J. Schoelkopf, Life after charge noise: Recent results with transmon qubits, Quantum Inf. Process. 8, 105 (2009).

[9] J. Koch, T. M. Yu, J. Gambetta, A. A. Houck, D. I. Schuster, J. Majer, A. Blais, M. H. Devoret, S. M. Girvin, and R. J. Schoelkopf, Charge-insensitive qubit design derived from the Cooper pair box, Phys. Rev. A 76, 042319 (2007).

[10] M. Brink, J. M. Chow, J. Hertzberg, E. Magesan, and S. Rosenblatt, in 2018 IEEE International Electron Devices Meeting (IEDM) (IEEE, San Francisco, 2018), p. 6.

[11] W. C. Smith, A. Kou, X. Xiao, U. Vool, and M. H. Devoret, Superconducting circuit protected by two-Cooper-pair tunneling, arXiv:1905.01206 (2019).

[12] A. Kitaev, Protected qubit based on a superconducting current mirror, arXiv:cond-mat/0609441 (2006).

[13] M. T. Bell, J. Paramanandam, L. B. Ioffe, and M. E. Gershenson, Protected Josephson Rhombus Chains, Phys. Rev. Lett. 112, 167001 (2014).

[14] J. M. Dempster, B. Fu, D. G. Ferguson, D. I. Schuster, and J. Koch, Understanding degenerate ground states of a protected quantum circuit in the presence of disorder, Phys. Rev. B 90, 094518 (2014).

[15] A. Kou, W. C. Smith, U. Vool, R. T. Brierley, H. Meier, L. Frunzio, S. M. Girvin, L. I. Glazman, and M. H. Devoret, Fluxonium-Based Artificial Molecule with a Tunable Magnetic Moment, Phys. Rev. X 7, 031037 (2017).

[16] P. Brooks, A. Kitaev, and J. Preskill, Protected gates for superconducting qubits, Phys. Rev. A 87, 052306 (2013).

[17] P. Groszkowski, A. Di Paolo, A. L. Grimsmo, A. Blais, D. I. Schuster, A. A. Houck, and J. Koch, Coherence properties of the $0-\pi$ qubit, New J. Phys. 20, 043053 (2018).

[18] A. Di Paolo, A. L. Grimsmo, P. Groszkowski, J. Koch, and A. Blais, Control and coherence time enhancement of the 0- $\pi$ qubit, New J. Phys. 21, 043002 (2019).

[19] B. Douçot and L. B. Ioffe, Physical implementation of protected qubits, Rep. Prog. Phys. 75, 072001 (2012).

[20] B. Douçot and J. Vidal, Pairing of Cooper Pairs in a Fully Frustrated Josephson-Junction Chain, Phys. Rev. Lett. 88, 227005 (2002).

[21] A. R. Klots and L. B. Ioffe, Set of holonomic and protected gates on topological qubits for realistic quantum computer, arXiv:1907.04379 (2019).

[22] S. Gladchenko, D. Olaya, E. Dupont-Ferrier, B. Douçot, L. B. Ioffe, and M. E. Gershenson, Superconducting nanocircuits for topologically protected qubits, Nat. Phys. 5, 48 (2009).

[23] J. R. Friedman and D. V. Averin, Aharonov-Casher-Effect Suppression of Macroscopic Tunneling of Magnetic Flux, Phys. Rev. Lett. 88, 050403 (2002).

[24] M. T. Bell, W. Zhang, L. B. Ioffe, and M. E. Gershenson, Spectroscopic Evidence of the Aharonov-Casher Effect in a Cooper Pair box, Phys. Rev. Lett. 116, 107002 (2016).

[25] S. E. De Graaf, S. T. Skacel, T. Hönigl-Decrinis, R. Shaikhaidarov, H. Rotzinger, S. Linzen, M. Ziegler, U.
Hübner, H.-G. Meyer, V. Antonov, and O. V. Astafiev, Charge quantum interference device, Nat. Phys. 14, 590 (2018).

[26] I. M. Pop, I. Protopopov, F. Lecocq, Z. Peng, B. Pannetier, O. Buisson, and W. Guichard, Measurement of the effect of quantum phase slips in a Josephson junction chain, Nat. Phys. 6, 589 (2010).

[27] O. Astafiev, Yu. A. Pashkin, Y. Nakamura, T. Yamamoto, and J.-S. Tsai, Quantum Noise in the Josephson Charge Qubit, Phys. Rev. Lett. 93, 267007 (2004).

[28] I. V. Pechenezhskiy, R. A. Mencia, L. B. Nguyen, Y.H. Lin, and V. E. Manucharyan, Quantum dynamics of quasicharge in an ultrahigh-impedance superconducting circuit, arXiv:1907.02937 (2019).

[29] D. M. Pozar, Microwave Engineering (John Wiley \& Sons, Hoboken, 2009).

[30] W. Zhang, K. Kalashnikov, W.-S. Lu, P. Kamenov, T. DiNapoli, and M. E. Gershenson, Microresonators Fabricated from High-Kinetic-Inductance Aluminum Films, Phys. Rev. Appl. 11, 011003 (2019).

[31] H. Rotzinger, S. T. Skacel, M. Pfirrmann, J. N. Voss, J. Münzberg, S. Probst, P. Bushev, M. P. Weides, A. V. Ustinov, and J. E. Mooij, Aluminium-oxide wires for superconducting high kinetic inductance circuits, Supercond. Sci. Tech. 30, 025002 (2016).

[32] P. C. J. J. Coumou, E. F. C. Driessen, J. Bueno, C. Chapelier, and T. M. Klapwijk, Electrodynamic response and local tunneling spectroscopy of strongly disordered superconducting tin films, Phys. Rev. B 88, 180505(R) (2013).

[33] D. Niepce, J. Burnett, and J. Bylander, High Kinetic Inductance NbN Nanowire Superinductors, Phys. Rev. Appl. 11, 044014 (2019).

[34] P. Kamenov, W.-S. Lu, K. Kalashnikov, T. DiNapoli, M. T. Bell, and M. E. Gershenson, Granular aluminum meandered superinductors for quantum circuits, arXiv:1910.00996 (2019).

[35] L. Grünhaupt, M. Spiecker, D. Gusenkova, N. Maleeva, S. T. Skacel, I. Takmakov, F. Valenti, P. Winkel, H. Rotzinger, W. Wernsdorfer, et al., Granular aluminium as a superconducting material for high-impedance quantum circuits, Nat. Mater. 18, 816 (2019).

[36] M. H. Devoret, Quantum fluctuations in electrical circuits, Les Houches, Session LXIII 7 (1995), p. 351.

[37] T. M. Hazard, A. Gyenis, A. Di Paolo, A. T. Asfaw, S. A. Lyon, A. A. H. A. Blais, and A. A. Houck, Nanowire Superinductance Fluxonium Qubit, Phys. Rev. Lett. 122, 010504 (2019).

[38] G. Burkard, Circuit theory for decoherence in superconducting charge qubits, Phys. Rev. B 71, 144511 (2005).

[39] G. Ithier, E. Collin, P. Joyez, P. J. Meeson, D. Vion, D. Esteve, F. Chiarello, A. Shnirman, Yu. Makhlin, J. Schriefl, and G. Schon, Decoherence in a superconducting quantum bit circuit, Phys. Rev. B 72, 134519 (2005).

[40] V. E. Manucharyan, Ph.D. thesis, School Yale University, 2012.

[41] J. Aumentado, M. W. Keller, J. M. Martinis, and M. H. Devoret, Nonequilibrium Quasiparticles and 2e Periodicity in single-Cooper-Pair Transistors, Phys. Rev. Lett. 92, 066802 (2004) 
[42] D. Rainis and D. Loss, Majorana qubit decoherence by quasiparticle poisoning, Phys. Rev. B 85, 174533 (2012).

[43] L. Sun, L. DiCarlo, M. D. Reed, G. Catelani, L. S. Bishop, D. I. Schuster, B. R. Johnson, G. A. Yang, L. Frunzio, L. Glazman, M. H. Devoret, and R. J. Schoelkopf, Measurements of Quasiparticle Tunneling Dynamics in a BandGap-Engineered Transmon Qubit, Phys. Rev. Lett. 108, 230509 (2012).
[44] N. A. Court, A. J. Ferguson, R. Lutchyn, and R. G. Clark, Quantitative study of quasiparticle traps using the singleCooper-pair transistor, Phys. Rev. B 77, 100501(R) (2008).

[45] M. E. Gershenzon and M. I. Falei, Absolute negative resistance in tunnel junctions of nonequilibrium superconductors, Sov. Phys. JETP 67, 389 (1988).

[46] M. E. Gershenzon, V. N. Gubankov, and Yu. E. Zhuravlev, Quantum effects in two-dimensional superconducting films at $T>T_{c}$, Sov. Phys. JETP 58, 167 (1983). 